\title{
Effect of storage time and temperature on stability of bioactive compounds in aseptically packed beverages prepared from rose hips and sea buckthorn berries
}

\author{
Staffan C. Andersson ${ }^{1}$, Anders Ekholm², Eva Johansson ${ }^{1}$, Marie E. Olsson ${ }^{1}$, Ingegerd Sjöholm³ ${ }^{3}$ Lena Nyberg ${ }^{4}$, Annika \\ Nilsson ${ }^{5}$ and Kimmo Rumpunen ${ }^{2}$ \\ ${ }^{1}$ Swedish University of Agricultural Sciences, Department of Plant Breeding, P.O. Box 101, SE-230 53 Alnarp, Sweden \\ ${ }^{2}$ Swedish University of Agricultural Sciences, Department of Plant Breeding, Balsgård, Fjälkestadsvägen 459, SE-291 94 \\ Kristianstad, Sweden \\ ${ }^{3}$ Lund University, Department of Food Technology, Engineering and Nutrition, PO Box 124, SE-221 00 Lund, Sweden \\ ${ }^{4}$ Skånemejerier, R\&D Department, SE-205 03 Malmö, Sweden \\ ${ }^{5}$ Kiviks Musteri AB, SE-277 35 Kivik, Sweden \\ e-mail: Staffan.Andersson@slu.se
}

\begin{abstract}
Rose hips and sea buckthorn berries contain high amounts of beneficial bioactive compounds. In this study the content and stability of natural vitamins and antioxidants were investigated in two fruit beverages made from rose hip powder, sea buckthorn berry purée and white grape juice concentrate, and wheat germ oil in one of the beverages. Beverages were formulated, pasteurised and filled aseptically in Tetra Brik packages and stored up to 35 days at $4{ }^{\circ} \mathrm{C}$ or $22{ }^{\circ} \mathrm{C}$. Samples were analysed for the content of ascorbic acid, carotenoids, major phenolics, tocochromanols, total phenols and total antioxidant capacity as ferric reducing ability. The largest changes occurred during the first 5 days of storage, thereafter mainly ascorbic acid decreased. In the wheat germ oil supplemented beverage, $\alpha$-tocopherol was more rapidly degraded. Negative correlations between the decrease of some carotenoids and tocochromanols, and the increase of some phenolics, suggest relationships of redox reactions specific to the amount of oil supplemented. From the present study we can conclude that additions of oil to beverages should be carefully selected to avoid oxidations of bioactive compounds. Furthermore, packaging of the beverages should be carried out at higher temperatures and thereafter it should be stored at low temperatures $\left(4^{\circ} \mathrm{C}\right)$, which decreased oxidation of all bioactive compounds except ascorbic acid.
\end{abstract}

Key words: ascorbic acid, carotenoids, phenols, tocopherols, FRAP

\section{Introduction}

Fruits, berries and vegetables contain numerous nutrients and other phytochemicals of importance to human health. Berries that are rich in vitamins and phenolics can trigger the endogenous antioxidant defence, and thereby may prevent against various cancers and cardiovascular diseases. Health benefits from consumption of supplements containing specific and pure antioxidants have however been questioned, while consumption of fruits, berries and vegetables have been shown beneficial (Liu 2003). Thus, products based on blends of fruits and vegetables rich in natural beneficial phytochemicals rather than on synthetically produced supplements should be safe and healthy for consumption.

Sea buckthorn berries (Hippophae rhamnoides L.) and rose hips (Rosa sp.) contain high amounts of vitamins, especially ascorbic acid (vitamin C) and other bioactive compounds, such as carotenoids (including pro-vitamin A), tocochromanols with vitamin E activity, phenolics and fatty acids (Rösch et al. 2003, Andersson et al. 2008, 2009, 2011, 2012, Yang et al. 2011). These fruits also have a high total antioxidant capacity compared to most fruits and vegetables (Halvorsen et al. 2002, Müller et al. 2011). Therefore, these two fruits are of specific interest to be evaluated as natural components of fruit based beverages.

Processing methods and storage conditions can influence the content of beneficial compounds in various products. Stability of bioactive compounds has been shown in a number of studies to vary and change during food and beverage processing and storage, and one important aspect is known to be the storage temperature (Shimoni 2004, Marete et al. 2011). Ingredients can be added during processing to increase stability and bioavailability of certain compounds, as well as to enhance flavour and mouthfeel of the product. It is known that bioavailability of e.g. $\beta$-carotene, lycopene and other carotenoids can be increased by proper processing conditions and by addition of plant oils (Van het Hof et al. 2000). Tocochromanols and carotenoids are known to protect each other from 
oxidation (Munné-Bosch \& Alegre 2002, Krinsky \& Johnson 2005). Also, polyphenols may play an important role for the stability since the hydrophobicity of many polyphenols is intermediate between that of ascorbic acid and that of tocochromanols. Therefore, polyphenols have been proposed to act at water-lipid interfaces and may be involved in oxidation regeneration pathways with ascorbic acid and tocochromanols (Manach et al. 2004). Thus a combination of natural antioxidants could improve the keeping quality of a product.

The aim of this investigation was to evaluate the effect of storage time and storage temperature on the post processing quality of model fruit beverages based on sea buckthorn berries and rose hips, and specifically, the effect of using wheat germ oil, a natural source of vitamin $\mathrm{E}$, as a beverage quality enhancer. In order to understand the security of the quality of beverages, the changes in content of several important bioactive compounds, such as ascorbic acid, carotenoids, tocochromanols as well as total antioxidant capacity, total phenols and major single phenolics were evaluated during storage in 35 days at different temperatures. Furthermore, interaction between the bioactive compounds and their changes were evaluated over the storage time.

\section{Materials and methods}

\section{Ingredients}

Commercially available frozen sea buckthorn berries (Hippophae cv. 'Sunny', harvested in August 2006, Latvia) were kept frozen at $-20^{\circ} \mathrm{C}$ until processing (February 2007). The frozen berries were shortly washed in hot water and heated to $60^{\circ} \mathrm{C}$ before being processed into puree using a medium mesh $(0.7 \mathrm{~mm}$ ) strainer (Leonhardt $\mathrm{GmbH}$, Germany). The yield of sea buckthorn puree was about $85 \%$ of berry fresh weight. The sea buckthorn puree was stored frozen $\left(-20^{\circ} \mathrm{C}\right)$ in plastic containers until use. Commercially available dried and milled rose hip powder (origin Chile), and $100 \%$ cold pressed wheat germ oil, was obtained from Skånemejerier, Sweden. White grape juice concentrate was obtained from Kiviks Musteri AB, Sweden.

\section{Beverage formulation, processing, packaging and storage}

A beverage (denoted RS) was made from $20 \%$ (by weight) sea buckthorn puree, $1.5 \%$ rose hip powder, $13.4 \%$ white grape juice concentrate and water. Another beverage (denoted RSO) was made of the same ingredients but was supplemented with $1 \%$ (by weight) wheat germ oil. 300 I of each beverage were pasteurised $\left(95^{\circ} \mathrm{C}, 30 \mathrm{~s}\right)$ (alfa laval, wide gap heat exchanger), cooled down to $25^{\circ} \mathrm{C}$ and filled aseptically on Tetra Brik packages (200 ml) using the industrial production facility (TBA9) at Kiviks Musteri AB and then stored at $4{ }^{\circ} \mathrm{C}$ or $22^{\circ} \mathrm{C}$. Samples for biochemical analyses were taken on days $0,5,10,21$ and 35 ( 2 samples day $0+4$ times $\times 2$ beverages $\times 2$ temperatures $=$ in total 18 samples), except analyses of specific phenolics for which samples were taken only on days 0 , 10 and 35 ( 2 samples day $0+2$ times $\times 2$ beverages $\times 2$ temperatures $=$ in total 10 samples). Additionally samples for ascorbic acid analyses also included storage at $8^{\circ} \mathrm{C}$, and samples were taken on days $0-5,7,10,14,21,35,49$, 70 and 112 ( 2 samples day $0+13$ times $\times 2$ beverages $\times 3$ temperatures $=$ in total 80 samples).

\section{Preparation of samples}

At selected days, samples were taken and transferred to $-20{ }^{\circ} \mathrm{C}$, besides ascorbic acid samples for which $1 \mathrm{ml}$ was immediately stabilized with $9 \mathrm{ml}$ of $2 \%$ meta-phosphoric acid (Merck, Germany) before transfer to $-20{ }^{\circ} \mathrm{C}$. After final collection all samples (except for ascorbic acid) were lyophilised and milled to a fine powder for 5 seconds in a laboratory mill (Yellow line, A10, IKA-Werke, Germany). All analyses were made in technical triplicates.

\section{Analysis of ascorbic acid}

For ascorbic acid analysis samples were thawed, placed in ultrasonic bath for $10 \mathrm{~min}$., and centrifuged for $10 \mathrm{~min}$. at $8500 \mathrm{rpm}$ (Centronic, USA). The supernatant $(1 \mathrm{ml}$ ) was filtered with a syringe particle filter (glass/nylon 0.45 $\mu \mathrm{m}, 30 \mathrm{~mm}$ Cameo, Sorbent AB, Sweden) directly into a HPLC-vial. The analysis was made on a Shimadzu HPLCsystem consisting of a communication bus module (SCL 10A-VP), two pumps (LC 10AD) and an autosampler (SIL$10 \mathrm{~A})$, using an Allure organic acids column $(5 \mu \mathrm{m}), 150 \times 4.6 \mathrm{~mm}$, and a guard column $(5 \mu \mathrm{m}) 10 \times 4.00 \mathrm{~mm}$ (Restek, USA) for separation. For detection a variable SPD-10A UV-VIS detector set to $254 \mathrm{~nm}$ was used. The column temperature was set to $25^{\circ} \mathrm{C}$ using an external oven. The mobile phase consisted of a $50 \mathrm{mM}$ sodium phosphate (Fluka, Switzerland) buffer set to $\mathrm{pH}$ 2.8. The flow rate was $1 \mathrm{ml} \mathrm{min}^{-1}$ and $10 \mu \mathrm{l} \mathrm{sample} \mathrm{was} \mathrm{injected} \mathrm{into} \mathrm{the} \mathrm{HPLC}$ system. Between every 13 sample an ascorbic acid standard was injected to monitor any degradation of ascorbic acid during the analysis. The peak was identified by retention time of an ascorbic acid standard (Sigma-Aldrich, USA). Quantification was carried by peak area. 


\section{Analysis of carotenoids}

For the carotenoids analyses, $1 \mathrm{~g}$ lyophilised sample was extracted in $20 \mathrm{ml}$ ethanol (99.7\%):n-hexane in proportions 4:3 with $0.01 \%$ BHT (butylated hydroxytoluene), using an orbital shaker (Forma Scientific Inc., Merietta, Ohio, USA) in darkness at $4^{\circ} \mathrm{C}$ for $20 \mathrm{~h}$, and was thereafter centrifuged at $10000 \mathrm{~g}$ for $10 \mathrm{~min}$. The analyses were made on an Agilent 1100 HPLC-system (Agilent Technology, USA) equipped with a diode-array detector, according to methods described by Andersson et al. $(2009,2011)$. The eluent consisted of solvent A ( $80 \%$ acetonitrile, $15 \% \mathrm{MeOH}$, and $5 \%$ dichloromethane, v:v) and solvent B (30\% acetonitrile, $20 \% \mathrm{MeOH}$, and $50 \%$ dichloromethane, v:v), with a binary gradient 0\% B (0-2 min), 0-25\% B (2-15 min), 25-60\% B (15-17 min), 60-90\% B (17-29 min), 90\% B (29-39 $\mathrm{min}), 90-0 \%$ B (39-41 min), and 0\% B (41-47 min). The column used was a Phenomenex Synergi 4 $\mu \mathrm{m}$ Hydro-RP $80 \mathrm{~A}, 250 \times 4.60 \mathrm{~mm}$ equipped with a Security Guard C18 pre-column, and detection was carried out at $458 \mathrm{~nm}$. Data for carotenoids were evaluated using the Chemstation A09.03 software (Agilent Technology, USA). For quantification of all carotenoid samples, all-trans-B-carotene Type 1 (Sigma-Aldrich, USA) were used, which was prequantified by a spectrophotometric method according to (Chen \& Yang 1992). Identification of carotenoids was done by use of spectral data for external standards (all-trans forms of lutein, zeaxanthin, $\beta$-cryptoxanthin, lycopene; Extrasynthese, Genay, France) and from literature data (Britton et al. 1995, Khachik et al. 1989, RodriguezAmaya 2001), and compared with spectra of the samples. Esterified carotenoids were separated from carotenes and xanthophylls by saponification of trial samples according to methods by Granado et al. (2001), but only quantified as total esterified carotenoids. Non-identified carotenoids were quantified as carotene according to retention time in the chromatogram by identifying the retention times for other known xanthophylls and carotenes. In this method xanthophylls have shorter retention times than carotenes.

\section{Analysis of tocochromanols}

For the tocochromanols analyses the extractions were the same as for carotenoids. The analyses were performed isocratically on a D-6000 HPLC system (Merck-Hitachi, Japan) with a fluorescence detector, according to methods described by Panfili et al.(2003) with modifications (Andersson et al. 2008, 2012). The eluent consisted of a solvent mixture consisting of $97.3 \% n$-hexane: $1.8 \%$ ethyl acetate: $0.9 \%$ acetic acid (all v/v). The column used was a Phenomenex Luna 5 m silica (2) 100A, $250 \times 4.60$ mm with a Security Guard silica $4 \mathrm{~mm} \mathrm{~L} \times 3.0 \mathrm{~mm}$ pre-column. The injection volume was $20 \mu \mathrm{l}$ and detection was carried out at $290 \mathrm{~nm}$ excitation and $330 \mathrm{~nm}$ emission. Data for tocochromanols were evaluated using the D-7000 HSM 4.1 software (Merck-Hitachi). For quantification of all tocopherols and tocotrienols DL- $\alpha$-tocopherol 97\% (Alfa Aesar, Karlsruhe, Germany) was used. Identification of tocochromanols was done using retention times compared against $\alpha-, b-, \gamma^{-}$, and $\delta$-tocopherol standards (Calbiochem, Merck, Darmstadt, Germany) and tocotrienol data from the literature (Abidi 2000, Kamal-Eldin et al. 2000, Panfili et al. 2003). The biologically active vitamin $E$ content $\left(C_{E}\right)$ was estimated by the formula presented by Bramley et al. (2000):

$$
C_{\mathrm{E}}=C_{\alpha}+C_{b} \times 0.5+C_{\nu} \times 0.1+C_{\delta} \times 0.03+C_{\alpha 3} \times 0.3
$$

Where the content of: $C_{\alpha}=\alpha$-tocopherol, $C_{B}=b$-tocopherol, $C_{\gamma}=\gamma$-tocopherol, $C_{\delta}=\delta$-tocopherol, and $C_{\alpha 3}=$ $\alpha$-tocotrienol

\section{Analysis of total antioxidant capacity}

Analysis of total antioxidant capacity was performed according to the FRAP (ferric reducing ability of plasma) method of Benzie \& Strain (1996), though slightly modified. Thus $25 \mathrm{mg}$ of sample was extracted with $1 \mathrm{ml} 90 \%$ methanol (Merck, Germany) for 15 minutes in an ultrasonic bath (Bandelin, Sonorex, Germany). The sample was then centrifuged (Centronic, USA) for 10 minutes in 8500 r.p.m and the supernatant was transferred to a new vial for further use. $300 \mathrm{mM}$ acetate buffer at pH 3.6, $10 \mathrm{mM}$ TPTZ (2,4,6-tripyridyl-s-triazine, Fluka, Switzerland) in $40 \mathrm{mM} \mathrm{HCl}$, and $20 \mathrm{mM} \mathrm{FeCl}_{3}$ in distilled water was used as stock solutions to prepare the reagent by mixing 25 $\mathrm{ml}$ acetate buffer, $2.5 \mathrm{ml}$ TPTZ solution and $2.5 \mathrm{ml} \mathrm{FeCl}_{3}$-solution. $10 \mu \mathrm{l}$ of the sample was then added to $1 \mathrm{ml}-$ reagent kept at $37{ }^{\circ} \mathrm{C}$ and the absorbance was measured at $593 \mathrm{~nm}$ after 4 minutes using Shimadzu UV-2101 PC spectrophotometer (Japan). Quantification was made from a standard stock solution of $2 \mathrm{mM}$ ferrous sulphate (FeSO ${ }_{4} 7 \mathrm{H}_{2} \mathrm{O}$, Fluka, Switzerland) in water. 


\section{Analysis of total phenols}

For extraction of total phenols $50 \mathrm{mg}$ lyophilised and milled sample was extracted with $1 \mathrm{ml} 50 \%$ (v/v) acidified (0.05 M phosphoric acid) ethanol for 15 hours in darkness at $5{ }^{\circ} \mathrm{C}$ using an orbital shaker. The sample was then centrifuged and the clear supernatant was recovered for further analyses of total phenols and major single phenolics. The content of total phenols was measured by a spectrophotometer (Shimadzu UV-2101PC, Japan) using Folin-Ciocalteu reagent according to Singleton \& Rosst (1965). Gallic acid (GA) (Sigma-Aldrich, Germany) was used as a standard for quantification and absorbance was recorded at $765 \mathrm{~nm}$.

\section{Analysis of single phenolics}

Analyses of single phenolics were carried out by an API 150 EX Turbo lonspray mass spectrometer (Sciex, USA) according to slightly modified methods previously published by Salminen et al. (1999 and 2005). The HPLC system thus consisted of a Perkin Elmer 200 autosampler (USA), two Perkin Elmer LC-200 Micro pumps, a guard column LiChroCART 4-4 100 RP-18 (5 $\mu \mathrm{m}$ ) (Merck, Germany) and a column LiChroCART 75-4 Superspher 100 RP-18 (Merck, Germany). Injection volume was $8 \mu \mathrm{l}$. The HPLC separation took place at ambient temperature and elution was carried out at a flow rate of $1 \mathrm{ml} \mathrm{min} \mathrm{m}^{-1}$. The eluent consisted of solvent A ( $0.4 \%$ formic acid) and solvent B (acetonitrile, LiChrosolve, Merck, Germany). For separation the binary mobile gradient was 0\% B (0-3 min), 0-30\% (3-35 min), 30-40\% (35-38 min) and 40-0 \% (38-42.5 min). The eluent was split off to $0.3 \mathrm{ml} \mathrm{min}^{-1}$ before being introduced into the ESI-MS system. The mass spectrometer was operated in a negative mode. The nebulizer gas and the curtain gas were delivered at 9 and $121 \mathrm{~min}^{-1}$, respectively. Temperature was kept at $300{ }^{\circ} \mathrm{C}$ and the ion spray voltage was set to $-4000 \mathrm{~V}$. In the scan mode ions with $\mathrm{m} / \mathrm{z}$ from 125 to 960 were measured (during $1.5 \mathrm{~s})$ with the orifice plate declustering potential (DP), the focusing ring potential (FP) and the entrance potential (EP) set to $-35 \mathrm{~V},-220 \mathrm{~V}$ and $-8.5 \mathrm{~V}$, respectively. In the single ion mode (for measurement of specific masses) the dwell time was $110 \mathrm{~ms}$ and the interface lens voltages were optimized as follows. For $(+)$-catechin $(\mathrm{m} / z$ 289), (-)-epicatechin (EC) (m/z 289), epigallocatechin (EGC) $(\mathrm{m} / z$ 305.2), GA ( $\mathrm{m} / \mathrm{z} 169)$, isorhamnetin $(\mathrm{m} / z$ 315.2) and quercetin $(\mathrm{m} / \mathrm{z} 301) \mathrm{DP}$ was set to $-31 \mathrm{~V}$, FP to $-150 \mathrm{~V}$ and EP to $-4.0 \mathrm{~V}$. For isorhamnetin-3-O-glucoside $(\mathrm{I3G})(\mathrm{m} / \mathrm{z}$ 477.4), kaempferol-3-O-glucoside (K3G) ( $\mathrm{m} / z$ 447.3), proanthocyanidin monomer glycoside (PAMG) $(\mathrm{m} / z$ 451.2) and quercetin-3-O-galactoside (Q3G) ( $\mathrm{m} / z$ 463.4) DP was set to -31V, FP to -170 V and EP to -8.5 V. For isorhamnetin-3-O-rutinoside (I3R) ( $\mathrm{m} / z$ 623.5), proanthocyanidin dimer aglycone (PADA) $(\mathrm{m} / \mathrm{z} 577)$, proanthocyanidin dimer diglycoside (PADDG) ( $\mathrm{m} / \mathrm{z}$ 901), proanthocyanidin dimer monoglycoside (PADMG) $(\mathrm{m} / \mathrm{z} 739)$ and quercetin3-O-rutinoside (Q3R) ( $\mathrm{m} / \mathrm{z}$ 609.5) DP was set to $-46 \mathrm{~V}$, FP to $-210 \mathrm{~V}$ and EP to $-8.5 \mathrm{~V}$. For quantification authentic standards (Extrasynthese, France) were used except for the proanthocyanidins, which were quantified as catechin equivalents; Q3G as Q3R; and I3R as I3G.

\section{Statistical analysis}

After normality test a general linear model (GLM) analyses of variance were carried out using SAS software (SAS Inst. Inc. Cary, North Carolina, USA) with significant level for LSD of means of $p=0.05$. Pearson's correlations were calculated between means of all investigated compounds for each beverage ( $N=9$ except for phenolics where $N$ = 5 since initial sample day 0 was the same for both temperature regimes, and therefore only used once in the statistical analysis). Significant correlations are presented in text at $p<0.05$, and with $p$-values in Appendices 1-3. Coefficients of variation (CV) were calculated among triplicates of samples to monitor variation in sample preparation and analyses.

\section{Results and discussion}

\section{Analyses of bioactive compounds and accuracy of results}

All investigated compounds, except some phenolics, were significantly affected by storage temperature, beverage blend, and the interaction between temperature and blend (Table 1-4, Fig. 1). The CV was $0.35 \%$ for the ascorbic acid standard indicating high repeatability and no trend for degradation of ascorbic acid during analysis was noticed. The CV among technical triplicates of samples was in average $2.40 \%$ for ascorbic acid, 3.38-8.53\% for single phenolics, $4.32-7.80 \%$ for carotenoids, $1.87-7.17 \%$ for tocochromanols, $2.07 \%$ for FRAP and $2.42 \%$ for total phenols indicating high accuracy of sample preparation and analyses. 
S.C. Andersson et al. (2015) 24: 273-288

Table 1. Content of different carotenoids $\left(\mu \mathrm{g} \mathrm{g}^{-1} \mathrm{dw}\right)$ in pasteurised and aseptically packaged rose hip and sea buckthorn berry beverages (blend), without (RS) or supplemented with wheat germ oil (RSO) at different storage days at $4{ }^{\circ} \mathrm{C}$ or $22^{\circ} \mathrm{C}$

\begin{tabular}{|c|c|c|c|c|c|c|c|c|}
\hline Blend & Day & $\begin{array}{c}\text { Temp. } \\
\left({ }^{\circ} \mathrm{C}\right)\end{array}$ & Zeaxanthin ${ }^{\mathrm{ABC}}$ & $\begin{array}{l}\text { Carotenes } \\
\text { LycopeneABC }\end{array}$ & $\beta^{\mathrm{ABC}}$ & Total ${ }^{A B C}$ & $\begin{array}{c}\text { Esterified } \\
\text { carotenoids }{ }^{\mathrm{ABC}}\end{array}$ & $\begin{array}{c}\text { Total } \\
\text { carotenoids }^{\mathrm{ABC}}\end{array}$ \\
\hline \multirow[t]{5}{*}{ RS } & 0 & - & $3.8 \mathrm{c}$ & $6.7 \mathrm{a}$ & $9.5 \mathrm{a}$ & $16.2 \mathrm{a}$ & $47.1 \mathrm{a}$ & $67.1 \mathrm{a}$ \\
\hline & 5 & 4 & $4.2 \mathrm{ab}$ & $5.4 \mathrm{bc}$ & $9.4 \mathrm{a}$ & $14.8 \mathrm{abc}$ & $44.4 \mathrm{a}$ & $63.4 \mathrm{ab}$ \\
\hline & 10 & 4 & $4.0 \mathrm{bc}$ & $5.0 \mathrm{c}$ & $8.5 \mathrm{~b}$ & $13.5 \mathrm{c}$ & $45.6 \mathrm{a}$ & $63.0 \mathrm{ab}$ \\
\hline & 21 & 4 & $4.2 \mathrm{ab}$ & $4.9 \mathrm{c}$ & $9.4 \mathrm{a}$ & $14.3 \mathrm{bc}$ & $44.1 \mathrm{a}$ & $62.6 \mathrm{~b}$ \\
\hline & 35 & 4 & $4.3 \mathrm{a}$ & $6.1 \mathrm{ab}$ & $9.4 \mathrm{a}$ & $15.4 \mathrm{ab}$ & $45.3 \mathrm{a}$ & $65.0 \mathrm{ab}$ \\
\hline \multirow[t]{5}{*}{ RS } & 0 & - & $3.8 \mathrm{a}$ & $6.7 \mathrm{a}$ & $9.5 \mathrm{a}$ & $16.2 \mathrm{a}$ & $47.1 \mathrm{a}$ & $67.1 \mathrm{a}$ \\
\hline & 5 & 22 & $3.0 \mathrm{~b}$ & $4.3 \mathrm{~b}$ & $7.8 \mathrm{~b}$ & $12.1 \mathrm{c}$ & $32.5 \mathrm{c}$ & $47.6 \mathrm{c}$ \\
\hline & 10 & 22 & $3.2 \mathrm{~b}$ & $4.7 \mathrm{~b}$ & $7.7 \mathrm{~b}$ & $12.3 \mathrm{c}$ & $30.9 c$ & $46.4 \mathrm{c}$ \\
\hline & 21 & 22 & $3.4 \mathrm{~b}$ & $6.1 \mathrm{a}$ & $8.3 \mathrm{~b}$ & $14.4 \mathrm{~b}$ & $35.9 \mathrm{~b}$ & $53.7 \mathrm{~b}$ \\
\hline & 35 & 22 & $3.2 \mathrm{~b}$ & $4.1 \mathrm{~b}$ & $7.8 \mathrm{~b}$ & $11.9 \mathrm{c}$ & $31.6 \mathrm{c}$ & $46.7 c$ \\
\hline \multirow[t]{5}{*}{ RSO } & 0 & - & $7.5 \mathrm{a}$ & $5.0 \mathrm{a}$ & $11.5 \mathrm{a}$ & 16.5 a & $60.8 \mathrm{a}$ & $84.8 \mathrm{a}$ \\
\hline & 5 & 4 & $5.1 \mathrm{~b}$ & $3.0 \mathrm{~cd}$ & $9.9 a b$ & $12.9 \mathrm{~b}$ & $39.2 \mathrm{~b}$ & $57.3 \mathrm{~b}$ \\
\hline & 10 & 4 & $5.0 \mathrm{~b}$ & $3.4 \mathrm{c}$ & $9.1 \mathrm{~b}$ & $12.5 \mathrm{~b}$ & $40.7 \mathrm{~b}$ & $58.2 b$ \\
\hline & 21 & 4 & $4.6 \mathrm{~b}$ & $2.7 \mathrm{~d}$ & $8.8 \mathrm{~b}$ & $11.5 \mathrm{~b}$ & $35.7 \mathrm{~b}$ & $51.8 \mathrm{~b}$ \\
\hline & 35 & 4 & $5.0 \mathrm{~b}$ & $4.1 \mathrm{~b}$ & $8.9 \mathrm{~b}$ & $13.0 \mathrm{~b}$ & $38.3 \mathrm{~b}$ & $56.3 \mathrm{~b}$ \\
\hline \multirow[t]{5}{*}{ RSO } & 0 & - & $7.5 \mathrm{a}$ & $5.0 \mathrm{a}$ & $11.5 \mathrm{a}$ & $16.5 \mathrm{a}$ & $60.8 \mathrm{a}$ & $84.8 \mathrm{a}$ \\
\hline & 5 & 22 & $4.5 \mathrm{~b}$ & $2.6 \mathrm{bc}$ & $7.9 \mathrm{~b}$ & $10.5 \mathrm{~b}$ & $32.9 \mathrm{~b}$ & $47.8 \mathrm{~b}$ \\
\hline & 10 & 22 & $4.5 \mathrm{~b}$ & $3.0 \mathrm{~b}$ & $7.7 \mathrm{~b}$ & $10.7 \mathrm{~b}$ & $34.2 \mathrm{~b}$ & $49.4 \mathrm{~b}$ \\
\hline & 21 & 22 & $4.4 \mathrm{~b}$ & $2.2 \mathrm{c}$ & $7.5 \mathrm{~b}$ & $9.8 \mathrm{~b}$ & $30.8 \mathrm{~b}$ & $44.9 \mathrm{~b}$ \\
\hline & 35 & 22 & $4.8 \mathrm{~b}$ & $1.3 \mathrm{~d}$ & $8.1 \mathrm{~b}$ & $9.4 \mathrm{~b}$ & $35.1 \mathrm{~b}$ & $49.2 \mathrm{~b}$ \\
\hline
\end{tabular}

The results are presented as means, based on three replicates for each sample. ${ }^{A}$ in header indicates significant difference for the four different treatments (blend beverage and temperature) $(p<0.05) .{ }^{B}$ in header indicates significant difference between the storage temperatures $(p<$ $0.05)$. ${ }^{C}$ in header indicates significant difference between beverages RS and RSO $(p<0.05)$. Different letters within columns indicates significant differences within the blend $(p<0.05)$

Table 2. Content of different tocochromanols $\left(\mu \mathrm{g} \mathrm{g}^{-1} \mathrm{dw}\right)$ in pasteurised and aseptically packaged rose hip and sea buckthorn berry beverages (blend), without (RS) or supplemented with wheat germ oil (RSO) at different storage days at $4{ }^{\circ} \mathrm{C}$ or $22^{\circ} \mathrm{C}$.

\begin{tabular}{|c|c|c|c|c|c|c|c|c|c|}
\hline \multirow[t]{2}{*}{ Blend } & \multirow[t]{2}{*}{ Day } & \multirow{2}{*}{$\begin{array}{c}\text { Temp. } \\
\left({ }^{\circ} \mathrm{C}\right)\end{array}$} & \multicolumn{4}{|c|}{ Tocopherols } & \multicolumn{2}{|c|}{ Tocotrienols } & \multirow{2}{*}{$\begin{array}{l}\text { Vitamin } E \\
\text { activity }^{A B C}\end{array}$} \\
\hline & & & $\alpha^{A B C}$ & $\beta^{\mathrm{ABC} C^{*}}$ & $\gamma$ & $\delta^{A B C}$ & $\alpha^{\mathrm{ABC}}$ & $\delta^{A B C}$ & \\
\hline \multirow[t]{5}{*}{ RS } & 0 & - & $96.9 \mathrm{a}$ & n.d. & n.d. & $24.4 \mathrm{~b}$ & $26.6 \mathrm{a}$ & $12.8 \mathrm{ab}$ & 105.6 a \\
\hline & 5 & 4 & $99.0 \mathrm{a}$ & n.d. & n.d. & $27.8 \mathrm{a}$ & $25.8 a b$ & $11.7 \mathrm{~b}$ & 107.6 a \\
\hline & 10 & 4 & $98.4 \mathrm{a}$ & n.d. & n.d. & $25.0 \mathrm{~b}$ & $23.6 \mathrm{bc}$ & $13.6 \mathrm{a}$ & $106.3 \mathrm{a}$ \\
\hline & 21 & 4 & $99.6 \mathrm{a}$ & n.d. & n.d. & $25.4 \mathrm{~b}$ & $22.5 c$ & $13.5 \mathrm{a}$ & $107.1 \mathrm{a}$ \\
\hline & 35 & 4 & $99.1 \mathrm{a}$ & n.d. & n.d. & $27.9 \mathrm{a}$ & $25.6 a b$ & $12.5 a b$ & $107.7 \mathrm{a}$ \\
\hline \multirow[t]{5}{*}{ RS } & 0 & - & $96.9 \mathrm{a}$ & n.d. & n.d. & $24.4 a b$ & $26.6 \mathrm{a}$ & $12.8 \mathrm{ab}$ & 105.6 a \\
\hline & 5 & 22 & 88.6 bc & n.d. & n.d. & $21.7 c$ & $24.5 a b$ & $10.2 \mathrm{c}$ & 96.6 bc \\
\hline & 10 & 22 & 88.9 bc & n.d. & n.d. & $22.9 \mathrm{bc}$ & $25.8 a b$ & $13.5 \mathrm{a}$ & $97.3 \mathrm{bc}$ \\
\hline & 21 & 22 & $90.9 \mathrm{~b}$ & n.d. & n.d. & $25.0 \mathrm{a}$ & $25.1 \mathrm{ab}$ & $13.1 \mathrm{a}$ & $99.1 \mathrm{~b}$ \\
\hline & 35 & 22 & $86.9 \mathrm{c}$ & n.d. & n.d. & $22.4 \mathrm{c}$ & $23.8 \mathrm{~b}$ & $12.1 \mathrm{~b}$ & $94.7 \mathrm{c}$ \\
\hline \multirow[t]{5}{*}{ RSO } & 0 & - & 278.7 a & $25.6 \mathrm{a}$ & tr. & $36.8 \mathrm{a}$ & $19.5 \mathrm{~b}$ & $21.6 \mathrm{a}$ & 298.5 a \\
\hline & 5 & 4 & $186.1 \mathrm{~b}$ & $15.6 \mathrm{bc}$ & tr. & $26.1 \mathrm{~b}$ & $20.8 a b$ & $14.3 \mathrm{bc}$ & $200.9 \mathrm{~b}$ \\
\hline & 10 & 4 & $186.6 \mathrm{~b}$ & $17.4 \mathrm{~b}$ & tr. & $23.0 \mathrm{~b}$ & $20.2 \mathrm{~b}$ & $13.4 \mathrm{c}$ & 202.1 b \\
\hline & 21 & 4 & $182.4 \mathrm{~b}$ & $17.3 \mathrm{~b}$ & tr. & $23.5 \mathrm{~b}$ & $22.0 \mathrm{a}$ & $14.8 \mathrm{~b}$ & $198.3 \mathrm{~b}$ \\
\hline & 35 & 4 & $187.9 \mathrm{~b}$ & $14.2 \mathrm{c}$ & tr. & $25.3 \mathrm{~b}$ & $21.5 \mathrm{a}$ & $10.9 \mathrm{~d}$ & $202.2 \mathrm{~b}$ \\
\hline \multirow[t]{5}{*}{ RSO } & 0 & - & 278.7 a & 25.6 a & tr. & $36.8 \mathrm{a}$ & $19.5 \mathrm{a}$ & $21.6 \mathrm{a}$ & 298.5 a \\
\hline & 5 & 22 & $176.2 \mathrm{c}$ & $11.7 \mathrm{~cd}$ & tr. & $21.4 \mathrm{~b}$ & 20.7 a & $15.8 \mathrm{~b}$ & 188.9 c \\
\hline & 10 & 22 & $183.1 \mathrm{~b}$ & $14.2 \mathrm{bc}$ & tr. & $22.4 \mathrm{~b}$ & $18.9 \mathrm{a}$ & $13.0 \mathrm{c}$ & 196.5 b \\
\hline & 21 & 22 & $178.7 \mathrm{bc}$ & $15.0 \mathrm{~b}$ & tr. & $24.4 \mathrm{~b}$ & $20.3 \mathrm{a}$ & $12.9 \mathrm{c}$ & $193.0 \mathrm{bc}$ \\
\hline & 35 & 22 & $183.1 \mathrm{~b}$ & $10.4 \mathrm{~d}$ & tr. & $22.9 \mathrm{~b}$ & $18.6 \mathrm{a}$ & $15.8 \mathrm{~b}$ & $194.5 \mathrm{bc}$ \\
\hline
\end{tabular}

The results are presented as means, based on three replicates for each sample. ${ }^{A}$ in header indicate significant difference for the four different treatments (blend beverage and temperature) $(p<0.05) .{ }^{\mathrm{B}}$ in header indicates significant difference between the storage temperatures $(p<0.05)$. ${ }^{C}$ in header indicates significant difference between beverages RS and RSO $(p<0.05)$, *excluded n.d. Different letters within columns indicate significant differences within the blend $(p<0.05)$. n.d. $=$ not detected, tr. $=$ traces 
Due to lack of resources, we did not repeat the full experiment, meaning that we have no replicates of the whole procedure, making it impossible to judge whether we should have got the same results if we had repeated the whole experiment. However, the high accuracy of the measurements, the consistency of the results, with consistent trends of changes over time periods of measurements, there should be no risk of applying the results of this study in order to understand effects of storage time and temperature on bioactive compounds in aseptically packed beverages.

\section{Effect of blend}

In the initial stage, when the beverages were just produced, the content of several of the bioactive compounds were higher in the beverage with germ oil (RSO) compared to the beverage not containing germ oil (RS). Examples of such bioactive compounds which increased with the addition of germ oil are; zeaxanthin, esterified carotenoids, total carotenoids, all types of tocopherols, delta tocotrienols, and ascorbic acid (Tables 1-4, Fig. 1). Also, the vita$\min \mathrm{E}$ activity was higher in the blends with than without germ oil in the initially produced beverages (Table 2). For most of the other investigated compounds, not mentioned above, minor differences were noted in content between the different blends. As the blends were stored at different times and temperatures, the content of the bioactive compounds changed and in general, those compounds that were clearly higher in the RSO compared to the RS blends showed a faster decrease rate in the RSO blends as to in the RS blends (Table 1-4, Fig. 1).

The higher content of bioactive compounds and especially of the tocopherols in RSO as compared to RS can be explained by additionally tocopherols being present in the added oil. Further, it is well known that cereals, such as wheat, hold high contents of tocotrienols although the content might vary due to genotypic variation (Hussain et al. 2012). Wheat germ oil is known to be susceptible to oxidation due to its high content of unsaturated fatty acids (Sjövall et al. 2000). $\alpha$-Tocopherol can however inhibit this free radical chain reaction process (Frankel 1993). The high degradation rates of tocopherols and carotenoids in the RSO beverage could be explained by the oxidation processes from the added wheat germ oil, and possibly from berry carotenoids acting as pro-oxidants Also, tocotrienols were rapidly degraded or was not obtained from the wheat germ oil. Thus the composition and quality of the oil is an important aspect to consider when using it as a supplement in beverages.

\begin{tabular}{|c|c|c|c|c|}
\hline Blend & Day & $\begin{array}{c}\text { Temp. } \\
\left({ }^{\circ} \mathrm{C}\right)\end{array}$ & $\begin{array}{l}\text { Total phenols }{ }^{A B C} \\
\text { (mg GAE/g dw) }\end{array}$ & $\begin{array}{c}\text { FRAPABC }^{\text {ABC }} \\
\left(\mathrm{mmol} \mathrm{Fe}{ }^{2+} / 100 \mathrm{~g} \mathrm{dw}\right)\end{array}$ \\
\hline \multirow[t]{5}{*}{ RS } & 0 & - & $12.9 \mathrm{c}$ & $12.0 \mathrm{bc}$ \\
\hline & 5 & 4 & $13.4 \mathrm{bc}$ & $12.1 \mathrm{bc}$ \\
\hline & 10 & 4 & $14.2 \mathrm{a}$ & $11.9 \mathrm{c}$ \\
\hline & 21 & 4 & $13.8 \mathrm{ab}$ & $12.7 \mathrm{a}$ \\
\hline & 35 & 4 & $13.6 \mathrm{abc}$ & $12.5 \mathrm{ab}$ \\
\hline \multirow[t]{5}{*}{ RS } & 0 & - & $12.9 \mathrm{~b}$ & $12.0 \mathrm{a}$ \\
\hline & 5 & 22 & $13.0 \mathrm{~b}$ & $12.0 \mathrm{a}$ \\
\hline & 10 & 22 & $13.7 \mathrm{a}$ & $12.3 \mathrm{a}$ \\
\hline & 21 & 22 & $13.8 \mathrm{a}$ & $12.5 \mathrm{a}$ \\
\hline & 35 & 22 & $13.4 a b$ & $12.1 \mathrm{a}$ \\
\hline \multirow[t]{5}{*}{ RSO } & 0 & - & $12.2 \mathrm{~b}$ & $11.5 \mathrm{a}$ \\
\hline & 5 & 4 & $12.4 \mathrm{~b}$ & $11.4 \mathrm{a}$ \\
\hline & 10 & 4 & $13.0 \mathrm{a}$ & $11.7 \mathrm{a}$ \\
\hline & 21 & 4 & $13.1 \mathrm{a}$ & $11.4 \mathrm{a}$ \\
\hline & 35 & 4 & $12.8 \mathrm{a}$ & $11.7 \mathrm{a}$ \\
\hline \multirow[t]{5}{*}{ RSO } & 0 & - & $12.2 \mathrm{~b}$ & $11.5 \mathrm{ab}$ \\
\hline & 5 & 22 & $13.1 \mathrm{a}$ & $11.2 \mathrm{~b}$ \\
\hline & 10 & 22 & $13.3 \mathrm{a}$ & $11.6 \mathrm{a}$ \\
\hline & 21 & 22 & $12.9 \mathrm{ab}$ & $11.4 a b$ \\
\hline & 35 & 22 & $12.6 a b$ & $11.4 \mathrm{ab}$ \\
\hline
\end{tabular}

$\overline{\text { The results are presented as means, based on three replicates for each sample. }{ }^{A}}$ in header indicates significant difference for the four different treatments (blend beverage and temperature) $(p<0.05) .{ }^{\mathrm{B}}$ in header indicates significant difference between the storage temperatures $(p<0.05)$. ${ }^{\mathrm{C}}$ in header indicates significant difference between beverages RS and RSO $(p<0.05)$. Different letters within columns indicates significant differences within the blend $(p<0.05)$. 
Table 4a. Content of single phenolics ( $\mu \mathrm{g} \mathrm{g}^{-1} \mathrm{dw}$ ) in pasteurised and aseptically packaged rose hip and sea buckthorn berry beverages (blend), without (RS) or supplemented with wheat germ oil (RSO) at different storage days at $4{ }^{\circ} \mathrm{C}$ or $22^{\circ} \mathrm{C}$

\begin{tabular}{|c|c|c|c|c|c|c|c|c|c|c|}
\hline Blend & Day & $\begin{array}{l}\text { Temp. } \\
\left({ }^{\circ} \mathrm{C}\right)\end{array}$ & Catechin ${ }^{\#}$ & $\begin{array}{c}\text { Epi- } \\
\text { catechin }^{A B C}\end{array}$ & $\begin{array}{l}\text { Epigallo- } \\
\text { catechin }^{\mathrm{ABC}}\end{array}$ & $\begin{array}{l}\text { Gallic } \\
\text { acid }^{A B C}\end{array}$ & $\begin{array}{l}\text { Iso- } \\
\text { rham }{ }^{A B C}\end{array}$ & $\begin{array}{l}\text { Isorham-3 } \\
\text {-O-rut }{ }^{c^{* *} \not}\end{array}$ & $\begin{array}{l}\text { Isorham-3 } \\
\text {-O-gluc\% }\end{array}$ & $\begin{array}{l}\text { Kaempferol-3- } \\
\text { O-glucoside }\end{array}$ \\
\hline \multirow[t]{3}{*}{ RS } & 0 & 4 & $138.0 \mathrm{a}$ & $11.3 \mathrm{a}$ & $4.5 \mathrm{a}$ & $9.5 \mathrm{~b}$ & $2.5 \mathrm{a}$ & $93.7 \mathrm{a}$ & $80.5 \mathrm{a}$ & $9.6 \mathrm{a}$ \\
\hline & 10 & 4 & $145.3 \mathrm{a}$ & $11.9 \mathrm{a}$ & $4.6 \mathrm{a}$ & $10.0 \mathrm{~b}$ & $2.2 \mathrm{ab}$ & 100.4 a & $83.6 \mathrm{a}$ & $9.7 \mathrm{a}$ \\
\hline & 35 & 4 & $143.7 \mathrm{a}$ & $9.9 \mathrm{~b}$ & $4.1 \mathrm{~b}$ & $10.6 \mathrm{a}$ & $2.0 \mathrm{~b}$ & $101.3 \mathrm{a}$ & $85.8 \mathrm{a}$ & $10.5 \mathrm{a}$ \\
\hline \multirow[t]{3}{*}{ RS } & 0 & 22 & $138.0 \mathrm{a}$ & $11.3 \mathrm{a}$ & $4.5 \mathrm{a}$ & $9.5 \mathrm{~b}$ & $2.5 \mathrm{a}$ & $93.7 \mathrm{a}$ & $80.5 \mathrm{a}$ & $9.6 \mathrm{a}$ \\
\hline & 10 & 22 & $143.0 \mathrm{a}$ & $8.8 \mathrm{~b}$ & $4.2 \mathrm{~b}$ & $11.0 \mathrm{a}$ & $1.9 \mathrm{~b}$ & $102.0 \mathrm{a}$ & $83.8 \mathrm{a}$ & $10.0 \mathrm{a}$ \\
\hline & 35 & 22 & $129.8 \mathrm{a}$ & $6.8 c$ & $3.6 \mathrm{c}$ & $11.2 \mathrm{a}$ & $2.4 \mathrm{a}$ & $102.7 \mathrm{a}$ & 80.9 a & $10.2 \mathrm{a}$ \\
\hline \multirow[t]{3}{*}{ RSO } & 0 & 4 & $131.2 \mathrm{a}$ & $10.0 \mathrm{a}$ & $4.2 \mathrm{a}$ & $8.6 \mathrm{~b}$ & $1.3 \mathrm{a}$ & $88.4 \mathrm{a}$ & $75.4 \mathrm{a}$ & $9.0 \mathrm{a}$ \\
\hline & 10 & 4 & $136.5 \mathrm{a}$ & $10.3 \mathrm{a}$ & $4.6 \mathrm{a}$ & $8.8 a b$ & $1.2 \mathrm{a}$ & $95.5 \mathrm{a}$ & $82.6 \mathrm{a}$ & $9.0 \mathrm{a}$ \\
\hline & 35 & 4 & 139.5 a & $9.7 \mathrm{a}$ & $4.6 \mathrm{a}$ & $9.7 \mathrm{a}$ & $1.3 \mathrm{a}$ & $96.8 \mathrm{a}$ & $78.1 \mathrm{a}$ & $9.4 \mathrm{a}$ \\
\hline \multirow[t]{3}{*}{ RSO } & 0 & 22 & $131.2 \mathrm{a}$ & $10.0 \mathrm{a}$ & $4.2 \mathrm{ab}$ & $8.6 \mathrm{~b}$ & $1.3 \mathrm{a}$ & $88.4 \mathrm{a}$ & $75.4 \mathrm{a}$ & $9.0 \mathrm{a}$ \\
\hline & 10 & 22 & $140.7 \mathrm{a}$ & $8.4 \mathrm{~b}$ & $4.4 \mathrm{a}$ & $10.0 \mathrm{a}$ & $1.4 \mathrm{a}$ & $97.1 \mathrm{a}$ & $81.1 \mathrm{a}$ & $9.7 \mathrm{a}$ \\
\hline & 35 & 22 & $125.5 \mathrm{a}$ & $6.5 \mathrm{c}$ & $3.9 \mathrm{~b}$ & $10.2 \mathrm{a}$ & $1.3 \mathrm{a}$ & 94.9 a & $76.8 \mathrm{a}$ & $9.5 \mathrm{a}$ \\
\hline
\end{tabular}

The results are presented as means, based on three replicates for each sample. ${ }^{A}$ in header indicates significant difference for the four different treatments (blend beverage and temperature) $(p<0.05) .{ }^{B}$ in header indicates significant difference between the storage temperatures $(p<0.05)$. ${ }^{\mathrm{C}}$ in header indicates significant difference between beverages RS and RSO $(p<0.05)$. Different letters within columns indicate significant differences within the blend $(p<0.05)$. "Isorhamnetin 3-O-rutinoside is quantified as isorhamnetin 3-O-glucoside. "Catechin A: (a=RS $4^{\circ}, \mathrm{RS} 22^{\circ}, \mathrm{RSO} 4^{\circ}$; $\left.\mathrm{b}=\mathrm{RS} 22^{\circ}, \mathrm{RSO} 4^{\circ}, \mathrm{RSO} 22^{\circ}\right), \mathrm{B}:\left(\mathrm{a}=4^{\circ}, 20^{\circ}\right) \mathrm{C}:(\mathrm{a}=\mathrm{RS}, \mathrm{RSO}) .{ }^{\mathrm{r}}$ Isorhamnetin-3-O-

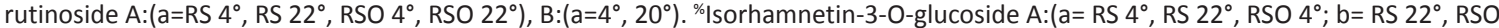
$4^{\circ}$, RSO $\left.22^{\circ}\right)$ B: $\left(a=4^{\circ}, 20^{\circ}\right) .{ }^{8}$ Kaempferol-3-O-glucoside A: $\left(a=\right.$ RS $4^{\circ}$, RS $22^{\circ} ; b=$ RSO $22^{\circ} ; c=$ RSO $\left.4^{\circ}\right)$. iso-rham = iso-rhamnetin; iso-rham-3O-rut = iso-rhamnetin-3-O-rutinoside; isorham-3-O-glu = isorhamnetin-3-O-glucoside

Table $4 \mathrm{~b}$. Content of phenolic compounds $\left(\mu \mathrm{g} \mathrm{g}^{-1} \mathrm{dw}\right.$ ) in pasteurised and aseptically packaged rose hip and sea buckthorn berry beverages (blend), without (RS) or supplemented with wheat germ oil (RSO) at different storage days at $4{ }^{\circ} \mathrm{C}$ or $22^{\circ} \mathrm{C}$

\begin{tabular}{|c|c|c|c|c|c|c|c|c|c|}
\hline Blend & Day & $\begin{array}{c}\text { Temp. } \\
\left({ }^{\circ} \mathrm{C}\right)\end{array}$ & $\begin{array}{c}\text { PA } \\
\text { monomer } \\
\text { glycoside }^{\mathrm{C}^{* \#}}\end{array}$ & $\begin{array}{l}\text { PA dimer } \\
\text { aglycone }{ }^{\mathrm{ABC} C^{*}}\end{array}$ & $\begin{array}{c}\text { PA dimer } \\
\text { mono- } \\
\text { glycoside }\end{array}$ & $\begin{array}{c}\text { PA dimer } \\
\text { diglycoside }{ }^{\mathrm{B}^{*} \%}\end{array}$ & Quercetin ${ }^{\mathrm{ABC}+}$ & $\begin{array}{l}\mathrm{Q}-3-\mathrm{O}- \\
\mathrm{gal}^{\mathrm{ABC}+}\end{array}$ & $\begin{array}{l}\text { Q-3-O- } \\
\text { rut }^{\mathrm{ABC}+}\end{array}$ \\
\hline \multirow[t]{3}{*}{ RS } & 0 & 4 & $67.2 \mathrm{a}$ & $12.1 \mathrm{a}$ & $113.6 \mathrm{a}$ & $96.3 \mathrm{a}$ & $74.2 \mathrm{a}$ & $79.2 \mathrm{a}$ & $29.5 \mathrm{a}$ \\
\hline & 10 & 4 & $72.3 \mathrm{a}$ & $13.2 \mathrm{a}$ & $128.3 \mathrm{a}$ & $102.8 \mathrm{a}$ & $74.2 \mathrm{a}$ & $80.3 \mathrm{a}$ & $28.8 \mathrm{a}$ \\
\hline & 35 & 4 & $68.5 \mathrm{a}$ & $13.5 \mathrm{a}$ & 126.9 a & $99.7 \mathrm{a}$ & 73.9 a & $83.0 \mathrm{a}$ & $32.6 \mathrm{a}$ \\
\hline \multirow[t]{3}{*}{ RS } & 0 & 22 & $67.2 \mathrm{a}$ & $12.1 \mathrm{a}$ & $113.6 \mathrm{~b}$ & $96.3 \mathrm{a}$ & $74.2 \mathrm{a}$ & $79.2 \mathrm{a}$ & $29.5 \mathrm{a}$ \\
\hline & 10 & 22 & $72.4 \mathrm{a}$ & $12.7 \mathrm{a}$ & $127.8 \mathrm{a}$ & $107.8 \mathrm{a}$ & $70.5 \mathrm{a}$ & 86.5 a & $30.0 \mathrm{a}$ \\
\hline & 35 & 22 & $69.8 \mathrm{a}$ & $11.8 \mathrm{a}$ & 130.9 a & $106.2 \mathrm{a}$ & $74.6 \mathrm{a}$ & 80.8 a & $32.3 \mathrm{a}$ \\
\hline \multirow[t]{3}{*}{ RSO } & 0 & 4 & $60.9 \mathrm{a}$ & $11.4 \mathrm{a}$ & 111.3 a & $89.0 \mathrm{a}$ & $65.9 \mathrm{a}$ & $71.9 \mathrm{a}$ & $24.4 \mathrm{a}$ \\
\hline & 10 & 4 & $67.7 \mathrm{a}$ & $12.6 \mathrm{a}$ & $114.1 \mathrm{a}$ & $97.2 \mathrm{a}$ & $63.3 \mathrm{a}$ & $72.7 \mathrm{a}$ & $25.6 \mathrm{a}$ \\
\hline & 35 & 4 & $67.2 \mathrm{a}$ & $12.1 \mathrm{a}$ & $121.5 \mathrm{a}$ & $98.8 \mathrm{a}$ & $63.6 \mathrm{a}$ & $77.5 a$ & $23.8 \mathrm{a}$ \\
\hline \multirow[t]{3}{*}{ RSO } & 0 & 22 & $60.9 \mathrm{~b}$ & $11.4 \mathrm{~b}$ & $111.3 \mathrm{~b}$ & $89.0 \mathrm{~b}$ & 65.9 a & 71.9 a & $24.4 \mathrm{a}$ \\
\hline & 10 & 22 & $70.7 \mathrm{a}$ & $12.2 \mathrm{a}$ & $127.8 \mathrm{a}$ & $115.6 \mathrm{a}$ & $61.9 \mathrm{a}$ & $75.6 \mathrm{a}$ & $25.4 \mathrm{a}$ \\
\hline & 35 & 22 & $67.2 \mathrm{ab}$ & $11.1 \mathrm{~b}$ & $130.6 \mathrm{a}$ & $105.3 \mathrm{a}$ & $63.0 \mathrm{a}$ & $77.6 \mathrm{a}$ & $25.1 \mathrm{a}$ \\
\hline
\end{tabular}

The results are presented as means, based on three replicates for each sample. ${ }^{A}$ in header indicates significant difference for the four different treatments (blend beverage and temperature) $(p<0.05) .{ }^{B}$ in header indicates significant difference between the storage temperatures $(p<0.05)$. ${ }^{C}$ in header indicates significant difference between blend beverages RS and RSO $(p<0.05)$. Different letters within columns indicates significant differences within the blend $(p<0.05) .{ }^{*} \mathrm{PA}=$ proanthocyanidin. PAs are quantified as catechin equivalents. ${ }^{+}$ Quercetin and quercetin-3-O-galactoside are quantified as quercetin-3-O-rutinoside. "PA monomer glycoside A: $\left(a=R S 22^{\circ}\right.$, RS $4^{\circ} ; b=R S 4^{\circ}$, RSO $22^{\circ} ; \mathrm{c}=$ RSO $\left.22^{\circ}, \mathrm{RSO} 4^{\circ}\right), \mathrm{B}:\left(\mathrm{a}=4^{\circ}, 20^{\circ}\right)$. ${ }^{\text {PA }}$ dimer monoglycoside $\mathrm{A}:\left(\mathrm{a}=\mathrm{RS} 22^{\circ}, \mathrm{RSO} 22^{\circ}, \mathrm{RS} 4^{\circ} ; \mathrm{b}=\mathrm{RSO} 4^{\circ}\right)$. ${ }^{\%} \mathrm{PA}$ dimer diglycoside A: $(\mathrm{a}$ $=$ RS $22^{\circ}$, RSO $22^{\circ}$, RS $^{\circ}$; $\mathrm{b}=$ RS $4^{\circ}$, RSO $\left.4^{\circ}\right)$ C:(a = RS, RSO). Q-3-O-gal = Quercetin-3-O-galactoside; Q-3-O-rut = Quercetin-3-O-rutinoside 


\section{Effect of storage time}

During the time of storage, the first days after pasteurization proved to be critical for changes in content of total and single carotenoids (Table 1), in content of tocopherols and delta tocotrienol, as well as in Vitamin E activity (Table 2). The largest decrease of the mentioned compounds occurred between day 0 and 5 and thereafter minor or no changes were noticed. The content of ascorbic acid decreased in both beverages at all storage temperatures during 112 days of storage. However, significant irregular changes occurred especially during the first week (Fig. 1). The total phenols, single phenolics and total antioxidant capacity showed minor irregular changes or a slightly increasing content (Table 3 and 4). The most obvious change was an increase in total phenols from day 0 to day 5 or 10 and thereafter no significant change was observed. The decrease of the mentioned compounds, carotenoids, tocopherols and ascorbic acid, over the storage time varied in relation to blend and storage temperature. In general, the decrease was larger for the RSO (in most cases from a higher initial value) and for the applied higher storage temperature (Table 1 and 2, Fig. 1).

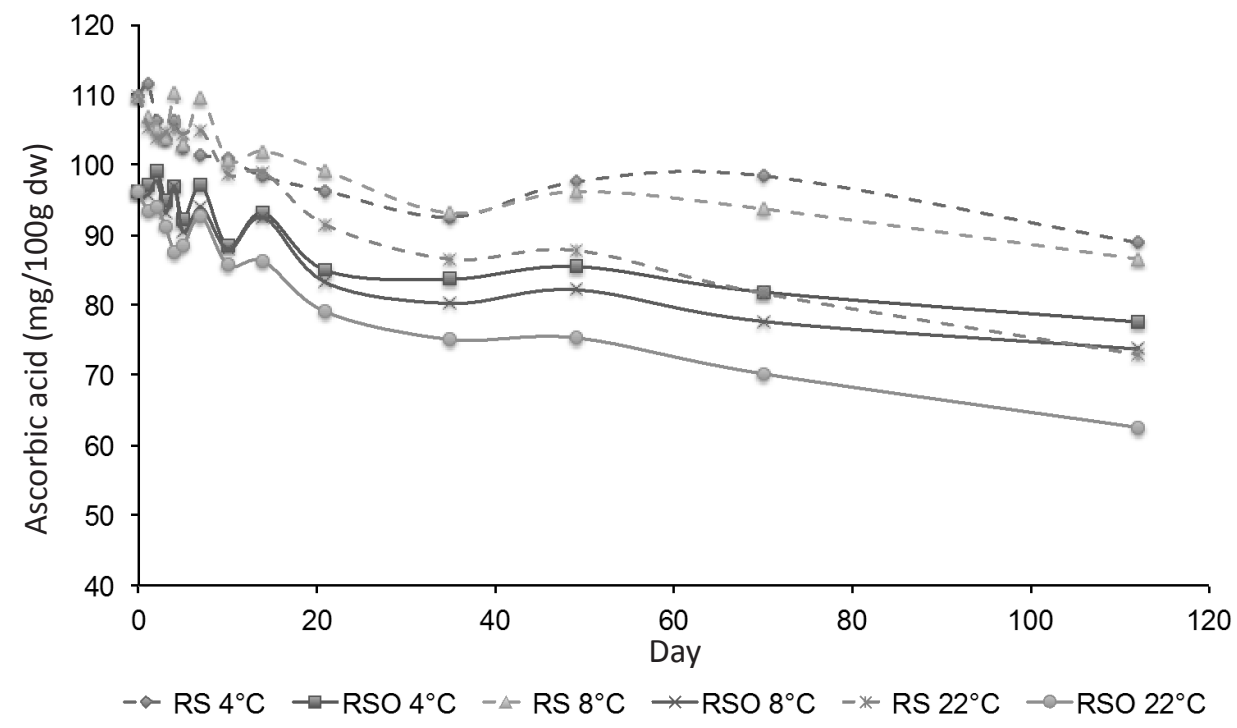

Fig. 1. Changes in content of ascorbic acid (mg $\left.100 \mathrm{~g}^{-1} \mathrm{dw}\right)$ during storage at $4{ }^{\circ} \mathrm{C}, 8{ }^{\circ} \mathrm{C}$ and $22{ }^{\circ} \mathrm{C}$ of a pasteurised and aseptically packaged rose hip and sea buckthorn berry beverage, without (RS) or with supplemented wheat germ oil (RSO). Results are means of triplicates. Average standard deviation = 2.21

It is known that content of bioactive compounds in beverages can be affected by processing, e.g. formulation, methods of mixing and pasteurization, as well as by temperature and time for storage. Some antioxidants, such as carotenoids, tocopherols, phenolics and ascorbic acid are known to interact through redox processes; tocochromanols and carotenoids may be regenerated by other antioxidants, such as ascorbic acid and phenolics (Niki et al. 1995, Kadoma et al. 2006), and ascorbic acid can also be regenerated by different phenolics (Amorati et al. 2002, 2003). After aseptic packaging, redox reactions may take place, especially during the initial storage period, due to mixing of different compounds. One possible explanation for the changes in content of bioactive compounds, especially during the first five days of storage, may be remnants of oxygen present from processing but the changes might also be influenced by other redox processes among different compounds in the beverage.

\section{Effect of storage temperature}

As mentioned above, the storage temperature influenced the decrease of bioactive compounds during the first five days, especially of those with initially high values (RSO compared to RS) and those making large changes. In those cases, the higher storage temperature $\left(20^{\circ} \mathrm{C}\right)$ led to increased changes compared to the lower storage temperature $4{ }^{\circ} \mathrm{C}$ (Table 1 and 2, Fig. 1). Thus, to preserve high levels of bioactive compounds (including antioxidants) in the beverage, a rapid cooling and storage at low temperature after packaging is advocated. Packaging at a higher temperature may however be preferable to get less oxygen in the beverage. Furthermore, storage in $20{ }^{\circ} \mathrm{C}$ led to lower content of carotenoids, tocochromanols, total phenolics, FRAP, ascorbic acid and some of the single phenolics after the full storage period as compared to when the beverages were stored at $20^{\circ} \mathrm{C}$ (Tables 1-4, Fig. 1). 


\section{Correlations}

Amongst the investigated compounds, a number of significant correlations were found (Appendices 1-3). A higher number of correlations among compounds were found in the beverages with added oil compared to without oil, especially for compounds having a stronger hydrophobic character with lower polarity i.e. the carotenoids and tocochromanols. The negative correlations found for total phenols on the one hand and zeaxantin, $\beta$-carotene, esterified carotenoids, total carotenoids, and $\delta$-tocopherol on the other hand in RSO, confirms the results from Graziani et al. (2003) showing that phenolics can protect tomato carotenoids, where the major part of the carotenoids are lycopene (Khachik et al. 2002). However, no correlation was found between phenolics and lycopene in RSO in the present study. Instead, a negative correlation was found for tocochromanols with phenolics in RSO, suggesting that some tocochromanols interact with phenolics in the oil phase, as previously suggested by Réblová \& Okrouhlá (2010). I3R and PAMG seemed to be the phenolics in interaction with most of carotenoids and tocochromanols in RSO. Also for RS negative correlations were found between PADDG and all carotenoids except for zeaxanthin. The revealed correlations between the mentioned phenolics and carotenoids suggest that the polarity of phenolics is a major factor contributing to the protection of carotenoids from oxidation. Phenolics have previously been shown to protect olive oil from oxidation during processing and storage (Baldioli et al. 1996) as well as to contribute to oil stability (Visioli \& Galli 1998). Further, it has been suggested that phenolics of olive oil can protect other antioxidants of tomatoes (Graziani et al. 2003) and that the bioavailability and absorption of tomato carotenoids can be increased by processing, including thermal heating and added olive oil (Stahl \& Sies 1992, Gärtner et al. 1997).

Furthermore, some phenolics in RSO, i.e. GA, PADMG and Q3G were seemingly interacting with ascorbic acid and to some extent also with $\beta$-tocopherol in the present study, these compounds were negatively correlated. These findings are supported by earlier investigations of interactions between ascorbic acid and phenolics (Amorati et al. 2002, 2003). The negative correlations between different phenolics may be due to a breakdown of glycosides and an increase of aglycones. Glycosides are usually more hydrophilic and the aglycones more hydrophobic. The lack of normal biosynthetic pathways due to pasteurization and reduced enzyme activity may be the reason for higher content of phenolics in RS as compared to RSO. However, the acid nature of the beverages can also contribute to cleavage of $O$-glycosides to aglycones and sugar through hydrolysis.

\section{Conclusions}

In fruit beverages of rose hip powder, sea buckthorn puree and grape juice concentrate, with or without supplemented wheat germ oil, pasteurized, packed aseptically and stored, a large change in presence of bioactive compounds occurred during the first 5 days of storage, but for ascorbic acid up to 35 days. Changes occurred faster at a high storage temperature. Notably the $\alpha$-tocopherol in the wheat germ oil supplemented beverage was more rapidly degraded as compared to $\alpha$-tocopherol in the non-supplemented beverage. Correlations between certain phenolic compounds and carotenoids suggest that the polarity of phenolics is a major factor contributing to the protection of carotenoids from oxidation.

\section{Acknowledgements}

SLU Partnerskap Alnarp, Kiviks Musteri och Skånemejerier is acknowledged for financial support and for providing pilot plant as well as commercial facilities for processing.

\section{References}

Abidi, S.L. 2000. Chromatographic analysis of tocol-derived lipid antioxidants. Journal of Chromatography A 881: 197-216.

Amorati, R., Ferroni, F., Lucarini, M., Pedulli, G.F. \& Valgimigli, L. 2002. A quantitative approach to the recycling of $\alpha$-tocopherol by coantioxidants. Journal of Organic Chemistry 67: 9295-9303.

Amorati, R., Ferroni, F., Pedulli, G.F. \& Valgimigli, L. 2003. Modeling the co-antioxidant behavior of monofunctional phenols. Applications to some relevant compounds. Journal of Organic Chemistry 68: 9654-9658.

Andersson, S.C., Olsson, M.E., Gustavsson, K.-E., Johansson, E. \& Rumpunen, K. 2012. Tocopherols in rose hips (Rosa spp.) during ripening. Journal of the Science of Food and Agriculture 92: 2116-2121.

Andersson, S.C., Olsson, M.E., Johansson, E. \& Rumpunen, K. 2009. Carotenoids in sea buckthorn (Hippophae rhamnoides L.) berries during ripening and use of pheophytin a as maturity marker. Journal of Agricultural and Food Chemistry 57: 250-258. 
Andersson, S.C., Rumpunen, K., Johansson, E. \& Olsson, M.E. 2008. Tocopherols and tocotrienols in sea buckthorn (Hippophae rhamnoides L.) berries during ripening. Journal of Agricultural and Food Chemistry 56: 6701-6706.

Andersson, S.C., Rumpunen, K., Johansson, E. \& Olsson, M.E. 2011. Carotenoid content and composition in rose hips (Rosa spp.) during ripening, determination of suitable maturity marker and implications for health promoting food products. Food Chemistry 128: 689-696.

Baldioli, M., Servili, M., Perretti, G. \& Montedoro, G.F. 1996. Antioxidant activity of tocopherols and phenolic compounds of virgin olive oil. Journal of the American Oil Chemists Society 73: 1589-1593.

Benzie, I.F.F. \& Strain, J.J. 1996. The ferric reducing ability of plasma (FRAP) as a measure of "Antioxidant power": The FRAP assay. Analytical biochemistry 239: 70-76.

Bramley, P.M., Elmadfa, I., Kafatos, A., Kelly, F.J., Manios, Y., Roxborough, H.E., Schuch, W., Sheehy, P.J.A. \& Wagner, K.H. 2000. Vitamin E. Journal of the Science of Food and Agriculture 80: 913-938.

Britton, G. 1995. UV/visible spectroscopy. In: Britton, G., Liaaen-Jensen, S. \& Pfander, H.E. (eds.). Carotenoids, vol. 1B: Spectroscopy. Basel, Switzerland: Birkhauser. p. 13-62.

Chen, B.H. \& Yang, S.H. 1992. An improved analytical method for the determination of carotenes and xanthophylls in dried plant materials and mixed feeds. Food Chemistry 40: 61-66.

Frankel, E.N. 1993. In search of better methods to evaluate natural oxidants and oxidative stability in food lipids. Trends in Food Science and Technology 4: 220-225.

Granado, F., Olmedilla, B. Gil-Martinez, E. \& Blanco, I. 2001. A fast reliable and low-cost saponification protocol for analysis of carotenoids in vegetables. Journal of Food Composition and Analysis 14: 479-489.

Graziani, G., Pernice, R., Lanzuise, S., Vitaglione, P., Anese, M. \& Fogliano, V. 2003. Effect of peeling and heating on carotenoid content and antioxidant activity of tomato and tomato-virgin olive oil systems. European Food Research and Technology 216: 116-121.

Gärtner, C., Stahl, W. \& Sies, H. 1997. Lycopene is more bioavailable from tomtato paste than from fresh tomatoes. American Journal of Clinical Nutrition 66: 116-122.

Halvorsen, B.L., Holte, K., Myhrstad, M.C.W., Barikmo, I., Hvattum, E., Fagertun Remberg, S., Wold, A.-B., Haffner, K., Baugerød, H., Frost Andersen, L., Moskaug, J. Ø., Jacobs, J.D.R. \& Blomhoff, R. 2002. A systematic screening of total antioxidants in dietary plants. The Journal of Nutrition 132: 461-471.

Hussain, A., Larsson, H., Olsson, M.E., Kuktaite, R., Grausgruber, H. \& Johansson, E. 2012. Is organically produced wheat a source of tocopherols and tocotrienols for health food? Food Chemistry 132: 1789-1795.

Kadoma, Y., Ishihara, M.I. \& Fujisawa, S. 2006. A quantitative approach to the free radical interaction between alpha-tocopherol and the coantioxidants eugenol, resveratrol or ascorbate. In vivo 20: 61-68.

Kamal-Eldin, A., Gorgen, S., Pettersson, J. \& Lampi, A.M. 2000. Normal phase high-performance liquid chromatography of tocopherols and tocotrienols: comparison of different chromatographic columns. Journal of Chromatography A 881: 217-227.

Khachik, F., Beecher, G.R. \& Lusby, W.R. 1989. Separation, identification, and quantification of the major carotenoids in extracts of apricots, peaches, cantaloupe, and pink grapefruit by liquid chromatography. Journal of Agricultural and Food Chemistry 37: 1465-1473.

Khachik, F., Carvalho, L., Bernstein, P.S., Muir, G.J., Zhao, D.-Y. \& Katz, N.B. 2002. Chemistry, distribution, and metabolism of tomato carotenoids and their impact on human health. Experimental Biology and Medicine 227: 845-851.

Krinsky, N.I. \& Johnson, E.J. 2005. Carotenoid action and their relation to health and disease. Molecular Aspects of Medicine 26 : 459-516.

Liu, R.H. 2003. Health benefits of fruit and vegetables are from additive and synergistic combinations of phytochemicals. The American Journal of Clinical Nutrition 78: 517S-20S.

Manach, C., Scalbert, A., Morand, C., Rémésy, C. \& Jiménez, L. 2004. Polyphenols: food sources and bioavailability. The American Journal of Clinical Nutrition 79: 727-747.

Marete, E.N., Jacquier, J.-C., O'Riordan, D. 2011. Feverfew as a source of bioactives for functional foods: Storage stability in model beverages. Journal of Functional Foods 3: 38-43.

Munné-Bosch, S. \& Alegre, L. 2002. The function of tocopherols and tocotrienols in plants. Critical Reviews in Plant Sciences 21 : 31-57.

Müller, L., Fröhlich, K. \& Böhm, V. 2011. Comparative antioxidant activities of carotenoids measured by ferric reducing antioxidant power (FRAP), ABTS bleaching assay (alpha TEAC), DPPH assay and peroxyl radical scavenging assay. Food Chemistry 129: 139-148.

Niki, E., Noguchi, N., Tsuchihashi, H. \& Gotoh, N. 1995. Interaction among vitamin-C, vitamin-E, and $\beta$-carotene. American Journal of Clinical Nutrition 62: 1322-1326.

Panfili, G., Fratianni, A. \& Irano, M. 2003. Normal phase high-performance liquid chromatography method for the determination of tocopherols and tocotrienols in cereals. Journal of Agricultural and Food Chemistry 51: 3940-3944.

Réblová, Z. \& Okrouhlá, P. 2010. Ability of phenolic acids to protect $\alpha$-tocopherol. Czech Journal of Food Science 28: 290-297.

Rodriguez-Amaya, D.B. 2001. A guide to carotenoid analysis in foods. Washington DC, USA: ILSI Press. 64 p.

Rösch, D., Bergmann, M., Knorr, D. \& Kroh, L.W. 2003. Structure antioxidant efficiency relationships of phenolic compounds and their contribution to the antioxidant activity of sea buckthorn juice. Journal of Agricultural and Food Chemistry 51: 4233-4239.

Salminen, J.-P., Ossipov, V., Loponen, J., Haukioja, E., \& Pihlaja, K. 1999. Characterisation of hydrolysable tannins from leaves of Betula pubescens by high-performance liquid chromatography-mass spectrometry. Journal of Chromatography A 864: $283-291$. 
Salminen, J.-P., Karonen, M., Lempa, K., Liimatainen, J., Sinkkonen, J., Lukkarinen, M. \& Pihlaja, K. 2005. Characterisation of proanthocyanidin aglycones and glycosides from rose hips by high-performance liquid chromatography-mass spectrometry, and their rapid quantification together with Vitamin C. Journal of Chromatography A 1077: 170-180.

Shimoni, E. 2004. Stability and shelf life of bioactive compounds during food processing and storage: Soy isoflavones. Journal of Food Science 69: R160-R166.

Singleton, V.L. \& Rosst, J.A. 1965. Colormetry of total phenolics with phosphomolybdic-phosphotungstick acid reagents. American Journal of Enology and Viticulture 16: 144-158.

Sjövall, O., Virtalaine, T., Lapveteläinen, A. \& Kallio, H. 2000. Development of rancidity in wheat germ analyzed by headspace gas chromatography and sensory analysis. Journal of Agricultural and Food Chemistry 48: 3522-3527.

Stahl, W. \& Sies, H. 1992. Uptake of lycopene and its geometrical isomers is greater from heat processed than from unprocessed tomato juice. Journal of Nutrition 122: 2161-2166.

Van het Hof, K.H., West, C.E., Weststrate, J.A. \& Hautvast, J.G.A.J. 2000. Dietary factors that affect the bioavailability of carotenoids. Journal of Nutrition 130: 503-506.

Visioli, F. \& Galli, C. 1998. Olive oil phenols and their potential effects on human health. Journal of Agricultural Food and Chemistry 46: 4292-4296.

Yang, B., Ahotupa, M., Määttä, P. \& Kallio, H. 2011. Composition and antioxidative activities of supercritical $\mathrm{CO}_{2}$-extracted oils from seeds and soft parts of northern berries. Food Research International 44: 2009-2017. 


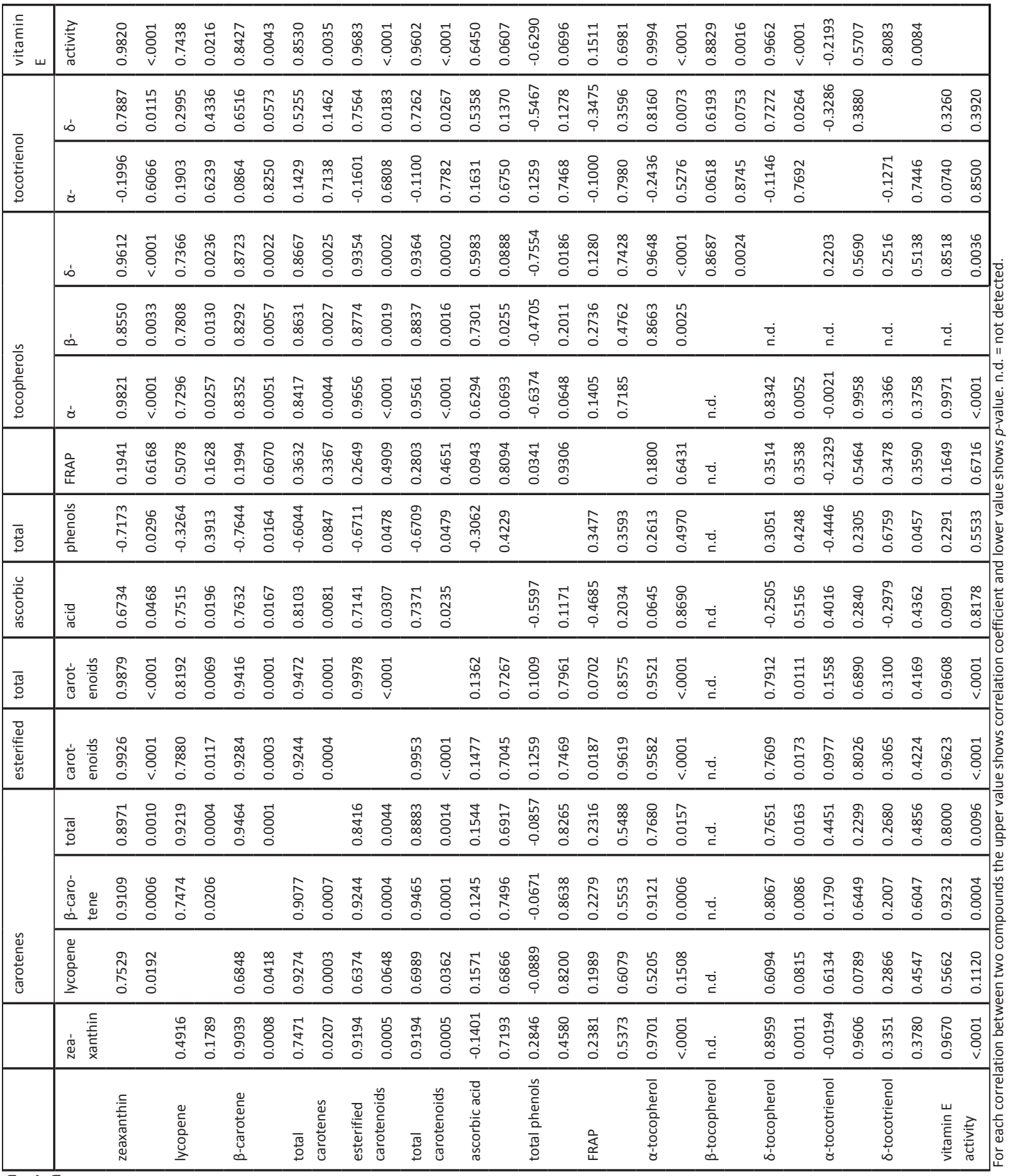

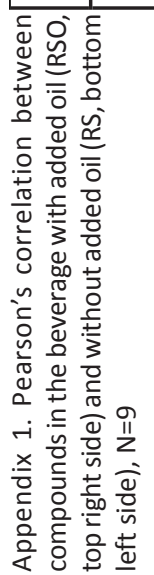




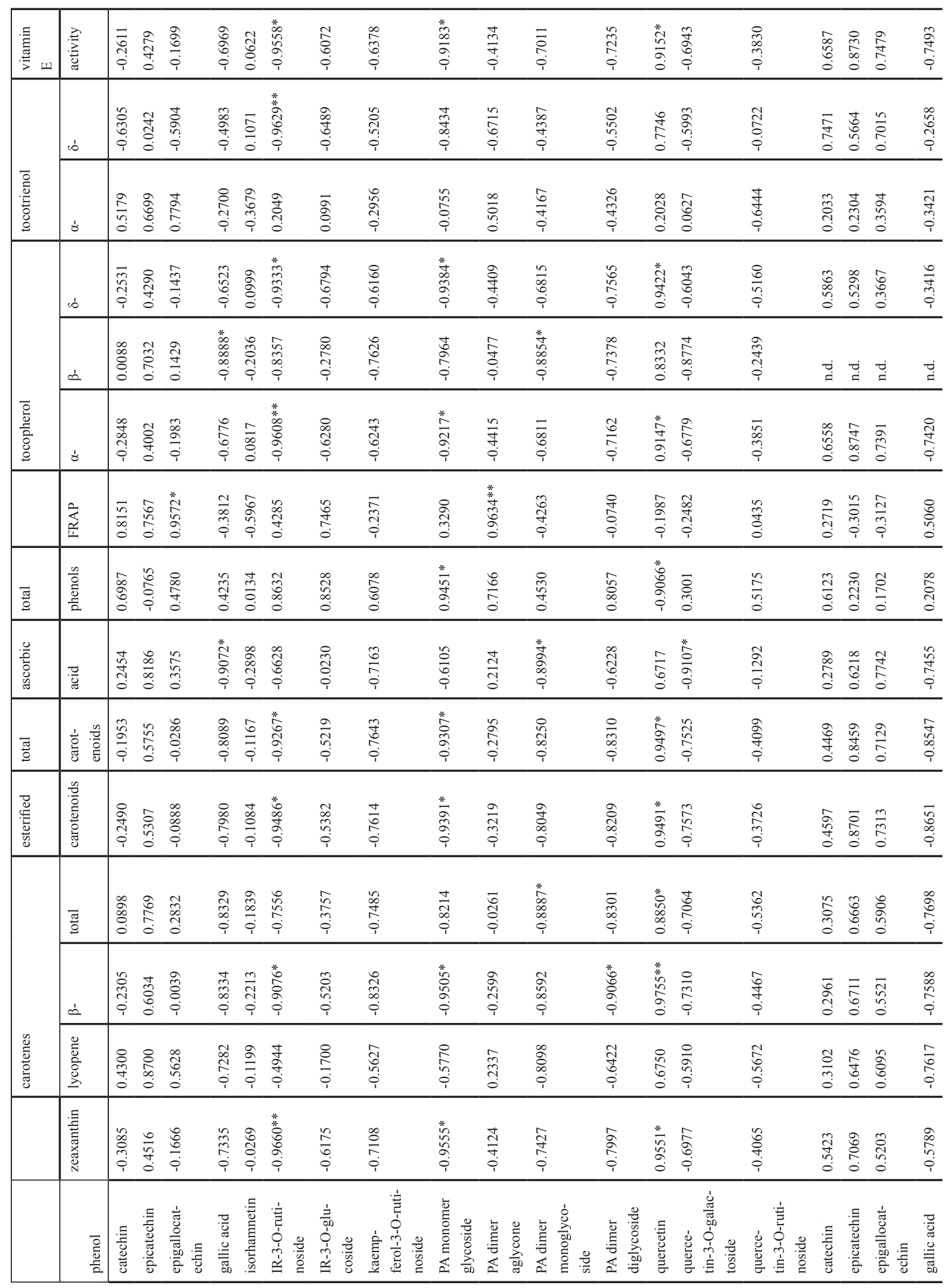

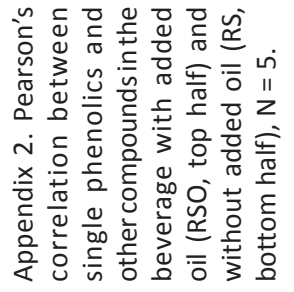




\begin{tabular}{|c|c|}
\hline 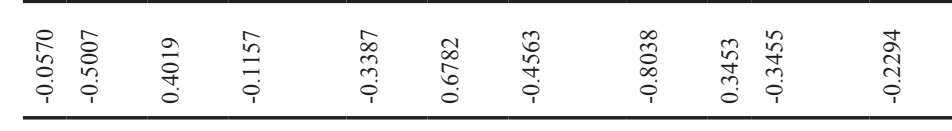 & \\
\hline 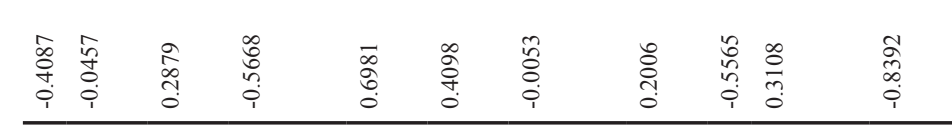 & \\
\hline 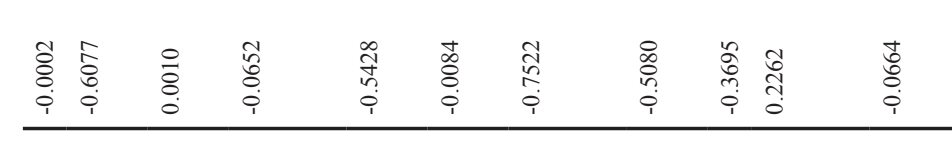 & \\
\hline 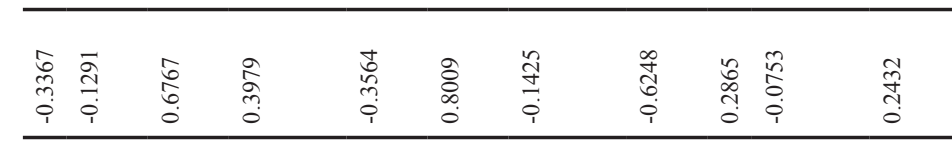 & \\
\hline 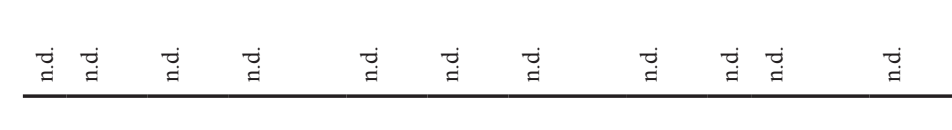 & \\
\hline 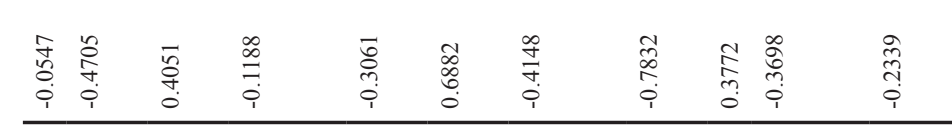 & \\
\hline 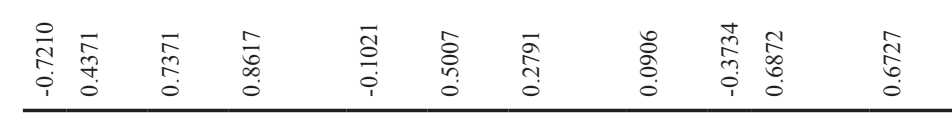 & \\
\hline 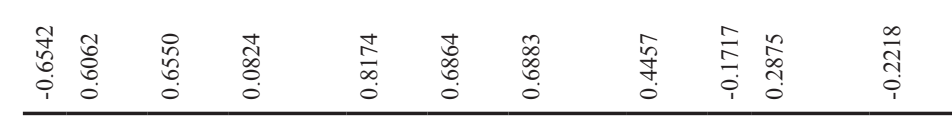 & \\
\hline 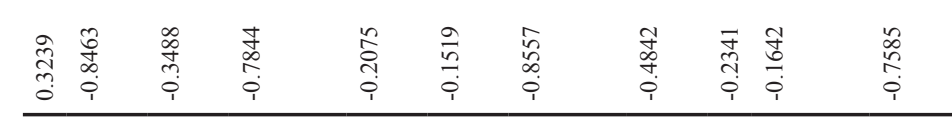 & \\
\hline 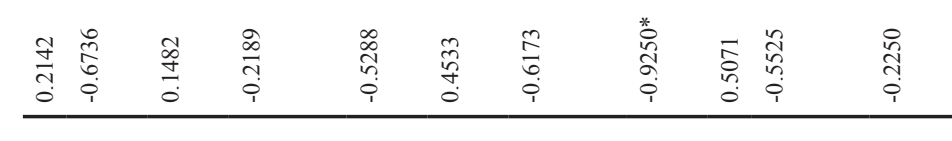 & \\
\hline 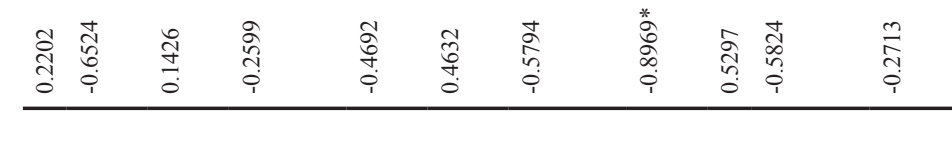 & \\
\hline 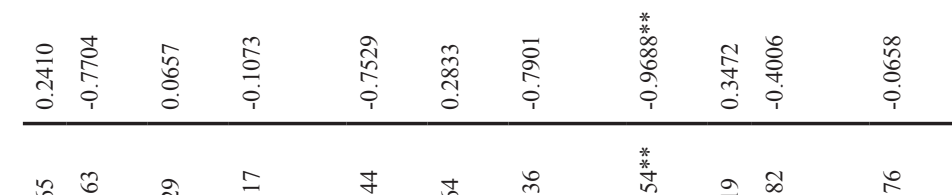 & \\
\hline
\end{tabular}

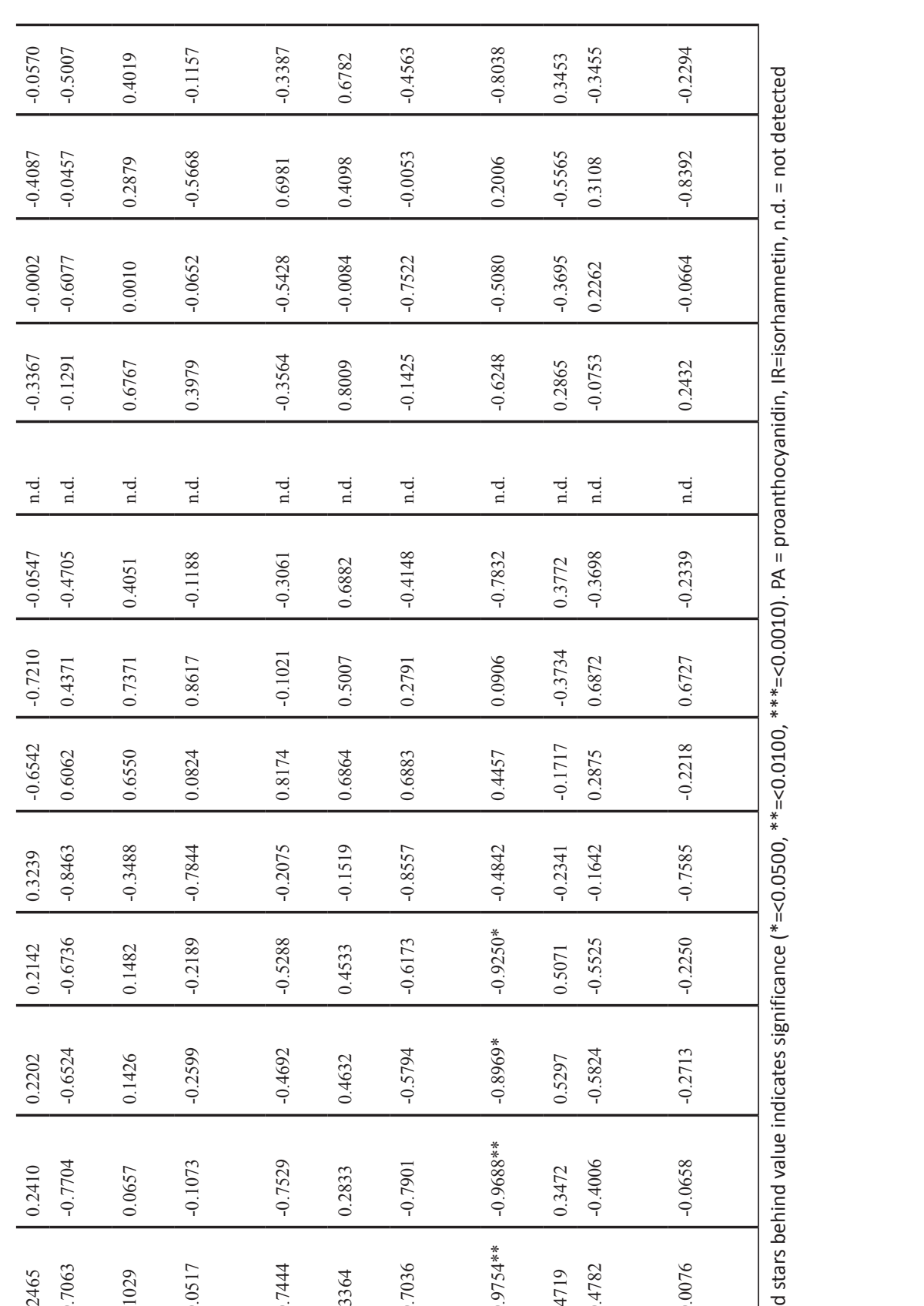

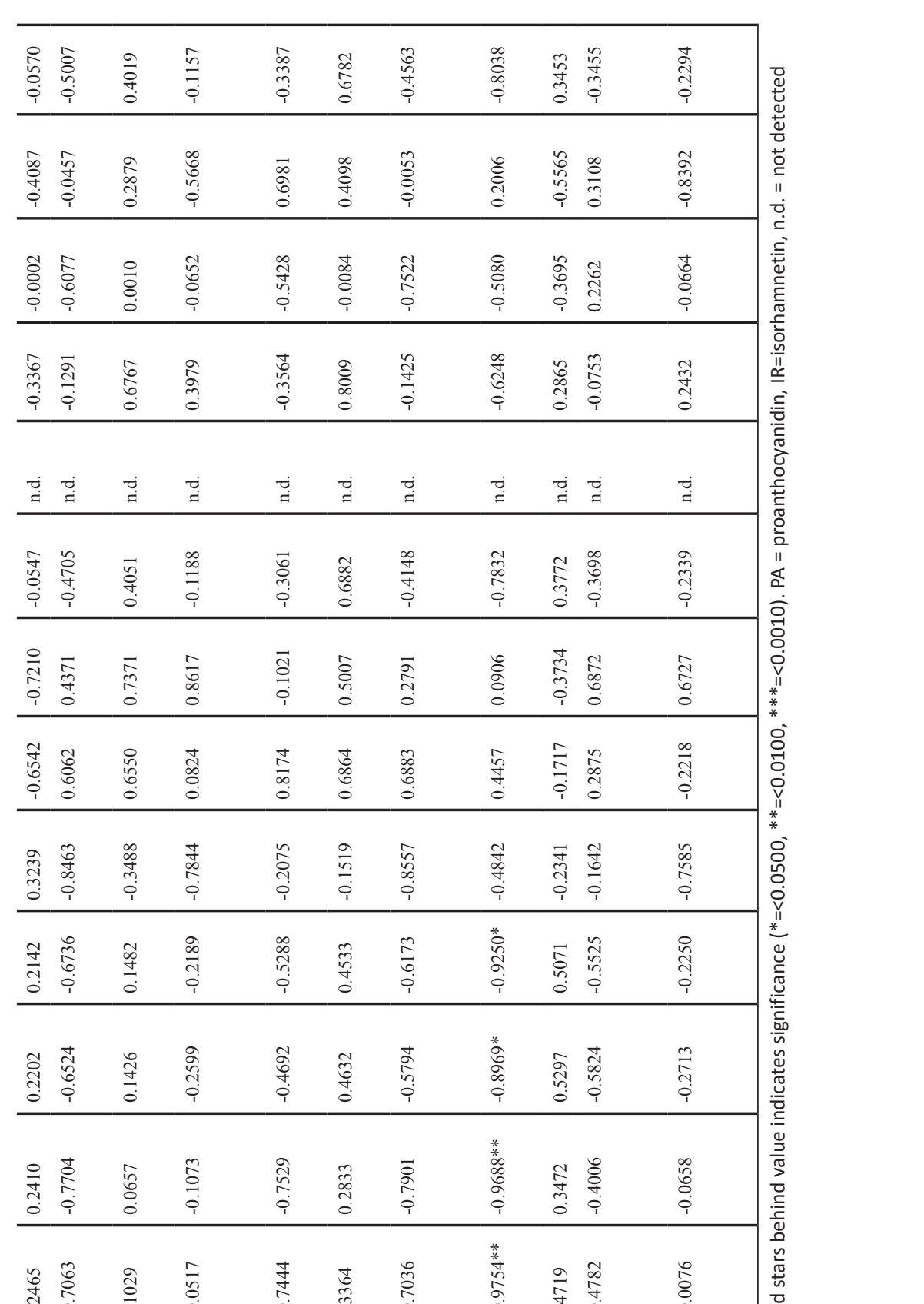

\begin{tabular}{|c|c|c|c|c|c|c|c|c|}
\hline 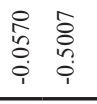 & $\begin{array}{l}0 \\
\text { ò } \\
0 \\
\end{array}$ & $\begin{array}{l}i n \\
\stackrel{n}{7} \\
i\end{array}$ & $\begin{array}{c}\hat{\infty} \\
\hat{0} \\
\hat{i} \\
i\end{array}$ & $\begin{array}{l}\infty \\
0 \\
0 \\
0 \\
0\end{array}$ & 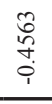 & $\begin{array}{c}\infty \\
0 \\
0 \\
0 \\
i \\
\end{array}$ & 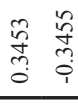 & $\begin{array}{l}\text { đ̃ } \\
\text { ते } \\
\text { î }\end{array}$ \\
\hline 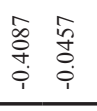 & 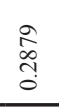 & $\begin{array}{l}\infty \\
: 0 \\
0 \\
0 \\
i \\
i \\
\end{array}$ & $\begin{array}{l}\bar{\alpha} \\
0 \\
0 \\
0\end{array}$ & $\begin{array}{l}\infty \\
\vdots \\
\vdots \\
0 \\
0 \\
\end{array}$ & $\begin{array}{l}\tilde{\hat{o}} \\
\stackrel{0}{i} \\
\dot{i}\end{array}$ & 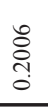 & 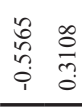 & $\begin{array}{c}\tilde{\alpha} \\
\infty \\
\infty \\
0 \\
\end{array}$ \\
\hline 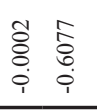 & $\begin{array}{l}0 \\
\stackrel{0}{0} \\
0 \\
0\end{array}$ & $\begin{array}{l}\tilde{\hat{o}} \\
\dot{0} \\
\dot{i}\end{array}$ & $\begin{array}{l}\infty \\
\text { 彳े } \\
0 \\
\vdots \\
\vdots \\
\end{array}$ & $\begin{array}{l}\text { a } \\
\text { o. } \\
\text { i } \\
\end{array}$ & $\begin{array}{l}\text { त̃ } \\
\hat{\kappa} \\
\hat{i}\end{array}$ & $\begin{array}{l}0 \\
0 \\
0 \\
0 \\
i \\
i\end{array}$ & $\begin{array}{cc}0 \\
0 \\
0 \\
0 \\
i \\
i\end{array}$ & $\begin{array}{l}\text { to } \\
\text { o. } \\
\dot{i}\end{array}$ \\
\hline 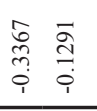 & $\begin{array}{l}5 \\
0 \\
0 \\
0 \\
0\end{array}$ & 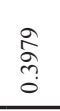 & $\begin{array}{c}\text { to } \\
\text { on } \\
\vdots \\
i \\
\end{array}$ & $\begin{array}{l}0 \\
\text { oे } \\
0 \\
0 \\
\end{array}$ & $\frac{n}{\stackrel{a}{+}}$ & 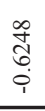 & 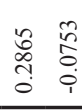 & 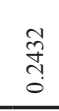 \\
\hline 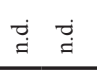 & $\stackrel{\rightleftarrows}{\dot{H}}$ & $\stackrel{\check{g}}{\dot{\leftrightarrows}}$ & 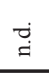 & $\stackrel{\check{I}}{\Xi}$ & $\stackrel{\check{\Xi}}{\exists}$ & $\stackrel{\check{J}}{ت}$ & 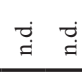 & $\stackrel{\dot{J}}{=}$ \\
\hline 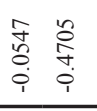 & $\begin{array}{l}\vec{r} \\
0 \\
\vdots \\
0 \\
\end{array}$ & 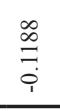 & 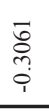 & $\begin{array}{l}\widetilde{1} \\
0 \\
0 \\
0 \\
0 \\
\end{array}$ & \begin{tabular}{l}
$\infty$ \\
\multirow{f}{*}{} \\
$i$ \\
$i$
\end{tabular} & $\begin{array}{l}\tilde{N} \\
\stackrel{\infty}{\infty} \\
\stackrel{i}{i} \\
\end{array}$ & 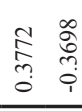 & $\begin{array}{l}\text { ते } \\
\text { तु } \\
\stackrel{i}{1}\end{array}$ \\
\hline 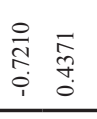 & $\begin{array}{l}\overrightarrow{0} \\
\hat{0} \\
0 \\
0\end{array}$ & $\begin{array}{l}\overline{\overrightarrow{0}} \\
\infty \\
0 \\
0\end{array}$ & $\begin{array}{l}\overrightarrow{\widehat{S}} \\
\stackrel{0}{i} \\
\end{array}$ & \begin{tabular}{l}
5 \\
\multirow{0}{0}{} \\
0 \\
0 \\
\end{tabular} & $\begin{array}{l}\overline{\hat{A}} \\
\text { ড̇ } \\
\end{array}$ & $\begin{array}{l}0 \\
\vdots \\
0 \\
0\end{array}$ & $\begin{array}{cc}c & 0 \\
0 & 0 \\
0 & 0 \\
i & 0 \\
\end{array}$ & $\begin{array}{l}\hat{E} \\
\vdots \\
0 \\
\end{array}$ \\
\hline $\begin{array}{ll}1 & 0 \\
0 & 0 \\
0 & 0 \\
0 & 0 \\
\end{array}$ & $\begin{array}{l}0 \\
\hat{0} \\
0 \\
0\end{array}$ & $\begin{array}{l}\tilde{U} \\
\stackrel{0}{0} \\
0 \\
0\end{array}$ & $\begin{array}{l}\frac{t}{\Delta} \\
\stackrel{\Delta}{\infty} \\
0 \\
0\end{array}$ & $\begin{array}{l}\text { to } \\
\text { o. } \\
0 \\
0\end{array}$ & 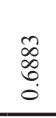 & $\begin{array}{l}5 \\
\text { s. } \\
\text { d. } \\
0\end{array}$ & 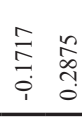 & 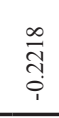 \\
\hline 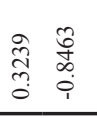 & 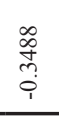 & 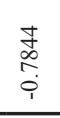 & $\begin{array}{l}\text { on } \\
\stackrel{i}{i} \\
i \\
i\end{array}$ & $\begin{array}{l}\frac{a}{n} \\
\text { ì } \\
\end{array}$ & $\begin{array}{l}\tilde{n} \\
0 \\
0 \\
i \\
i\end{array}$ & 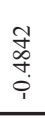 & 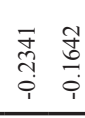 & $\begin{array}{l}\infty \\
\infty \\
0 \\
\hat{i} \\
i\end{array}$ \\
\hline 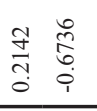 & $\underset{\substack{\tilde{d} \\
\\
0}}{0}$ & 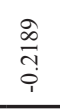 & 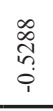 & 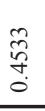 & $\begin{array}{l}m \\
\stackrel{5}{b} \\
\stackrel{i}{1} \\
\end{array}$ & $\begin{array}{l}\text { \% } \\
\text { à } \\
\text { ò } \\
\text { ì } \\
\end{array}$ & 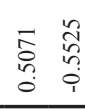 & $\begin{array}{l}\text { ते } \\
\text { तु } \\
\text { i } \\
\end{array}$ \\
\hline 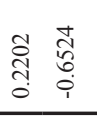 & 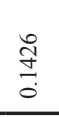 & 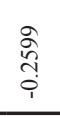 & 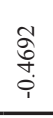 & $\begin{array}{l}\text { है } \\
\text { Ș } \\
\end{array}$ & $\begin{array}{l}\text { t. } \\
\vdots \\
\vdots \\
i \\
\end{array}$ & $\begin{array}{l}\text { *. } \\
\text { ôे } \\
\text { o. } \\
i \\
\end{array}$ & 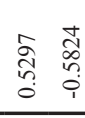 & $\begin{array}{l}\text { ât } \\
\text { ì } \\
i\end{array}$ \\
\hline 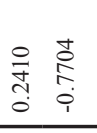 & 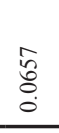 & 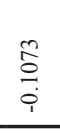 & $\begin{array}{l}\text { ह̂े } \\
\text { î } \\
\end{array}$ & 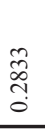 & $\begin{array}{l}\bar{o} \\
\vdots \\
\vdots \\
\end{array}$ & $\begin{array}{l}\text { *. } \\
\text { कo } \\
0 \\
0 \\
i \\
i \\
\end{array}$ & 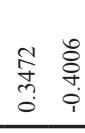 & $\begin{array}{l}\infty \\
0 \\
0 \\
0 \\
i\end{array}$ \\
\hline
\end{tabular}

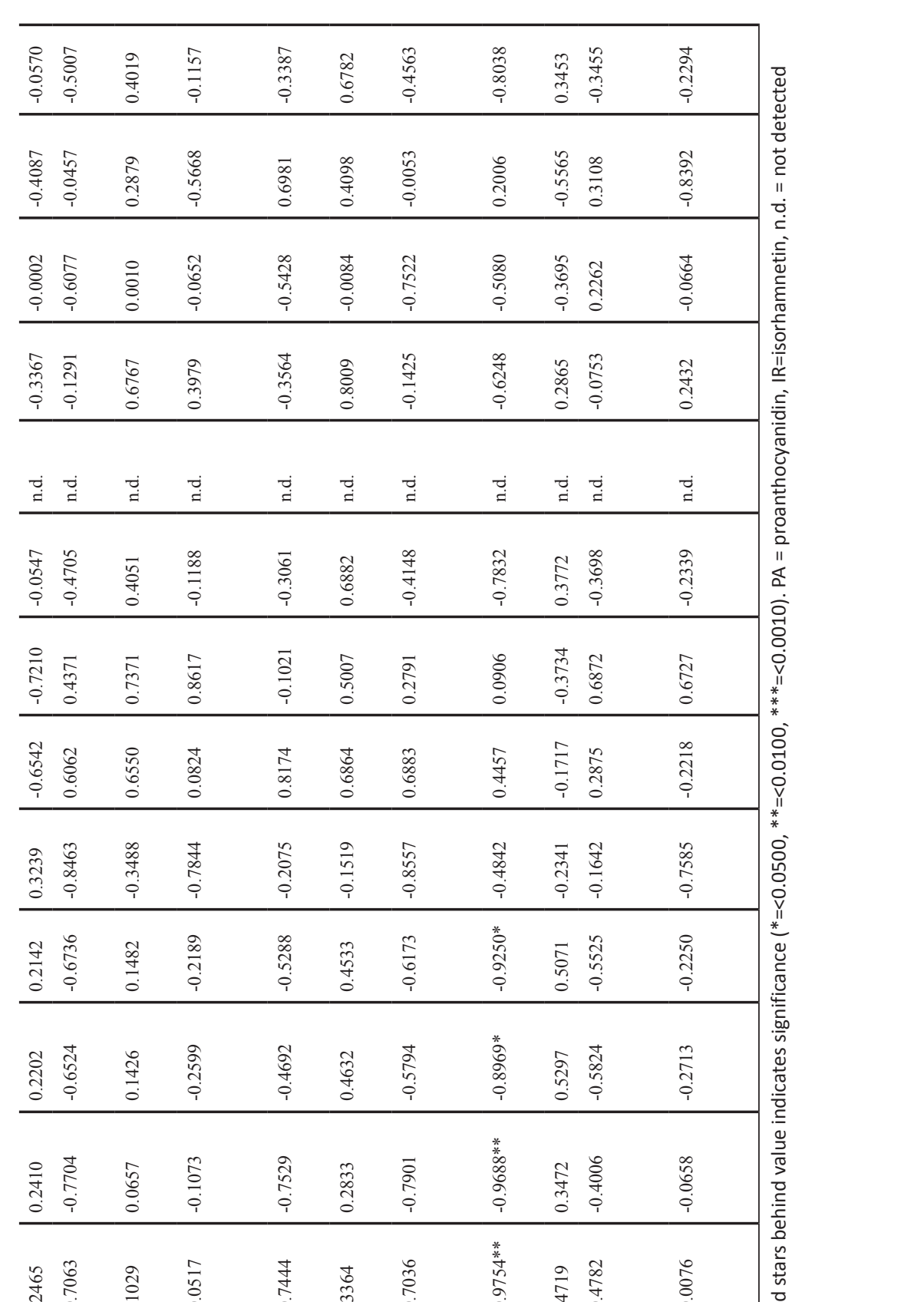

\begin{tabular}{|c|c|}
\hline 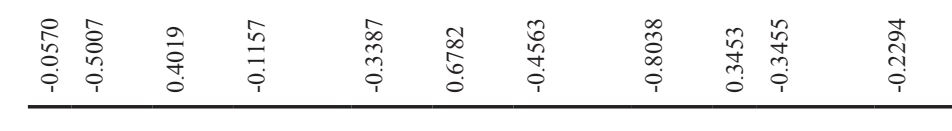 & \\
\hline 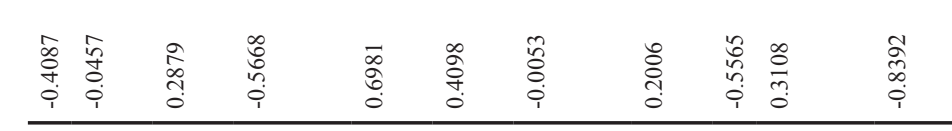 & \\
\hline 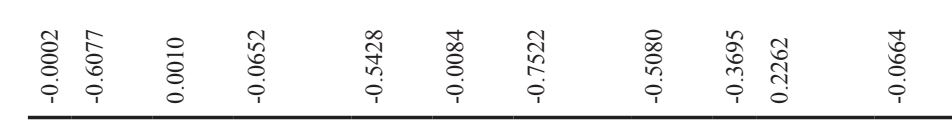 & \\
\hline 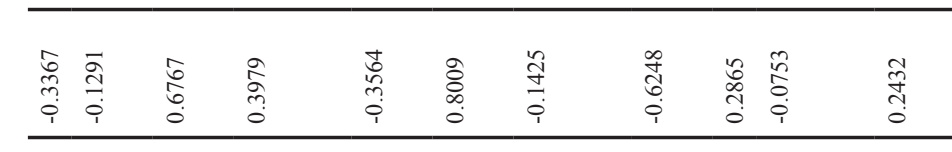 & \\
\hline 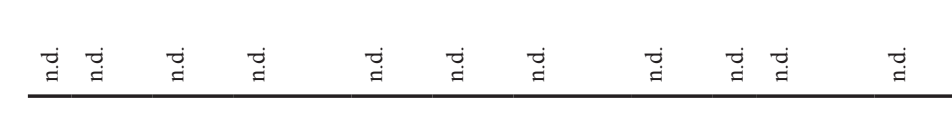 & \\
\hline 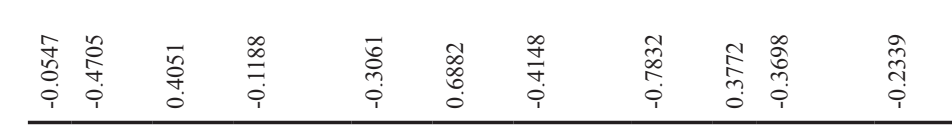 & \\
\hline 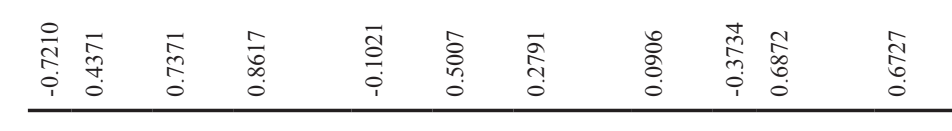 & \\
\hline 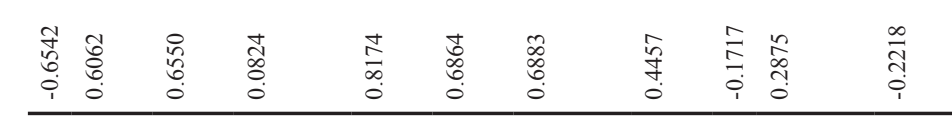 & \\
\hline 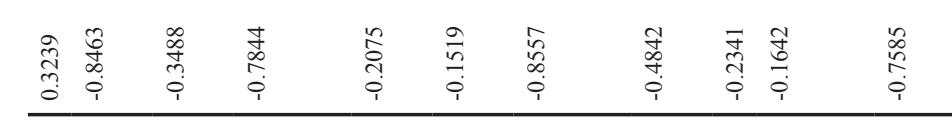 & \\
\hline 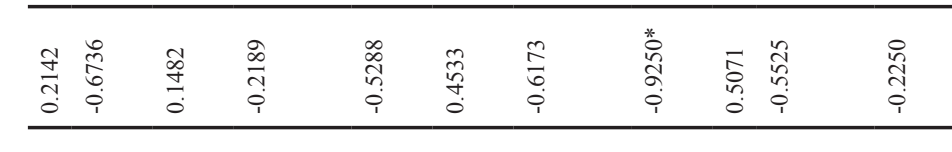 & \\
\hline 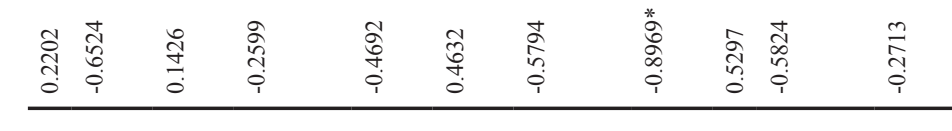 & \\
\hline 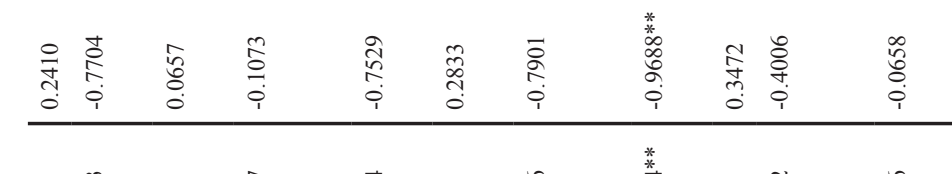 & \\
\hline
\end{tabular}

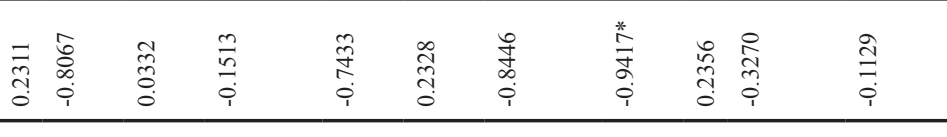

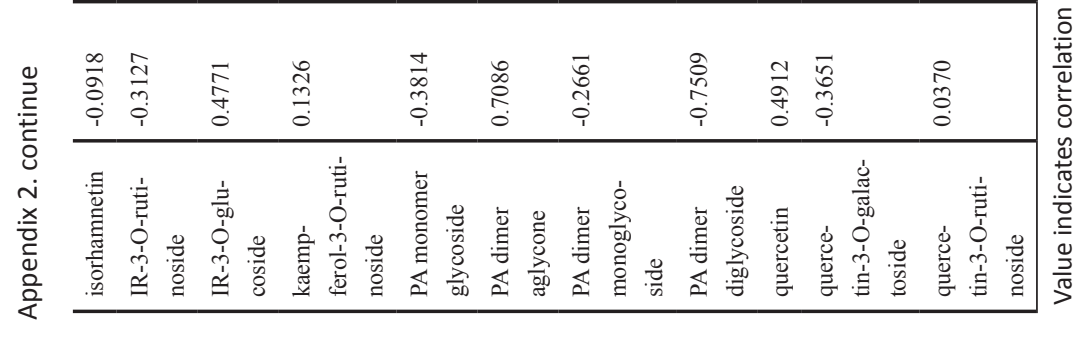




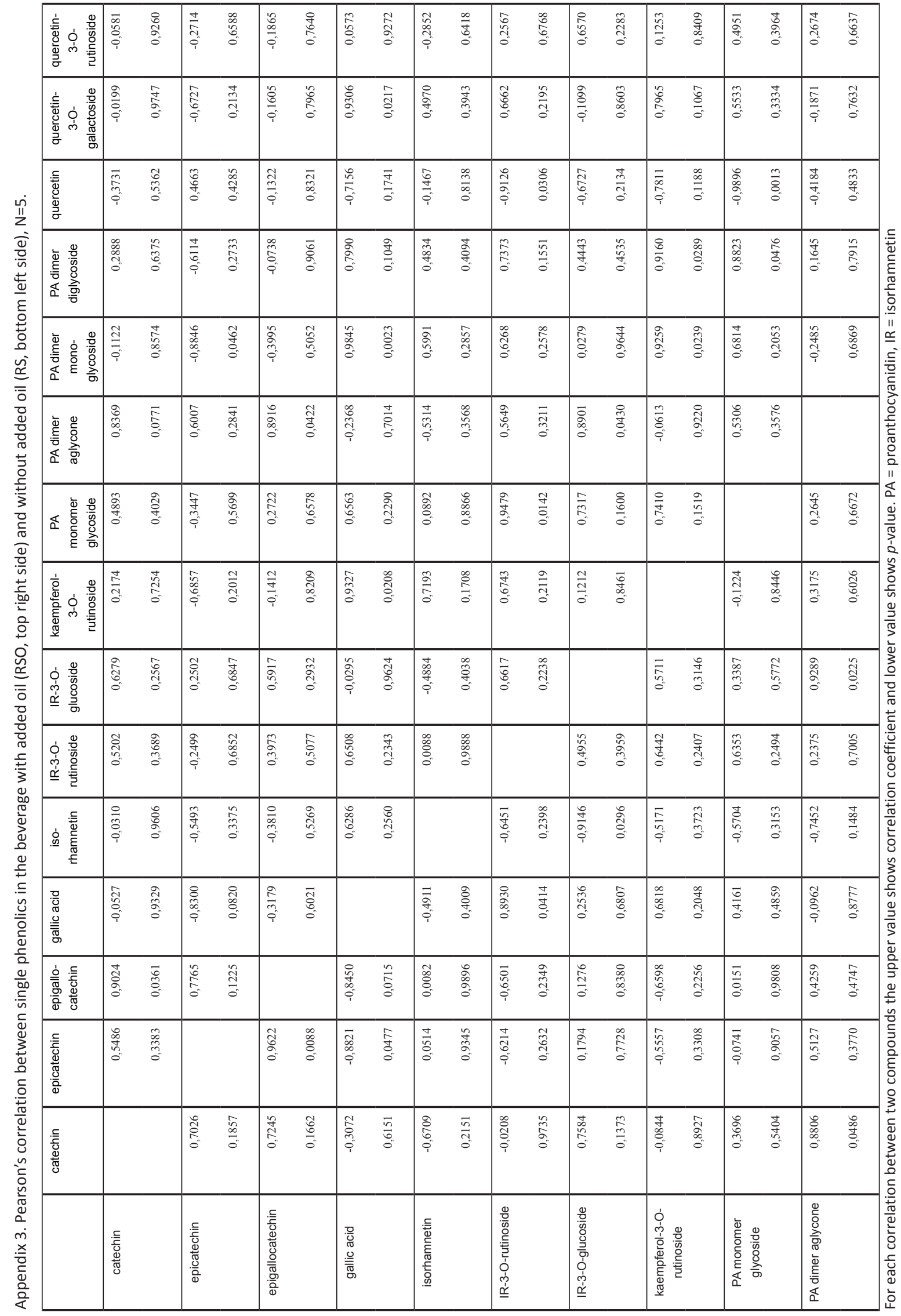




\begin{tabular}{|c|c|c|c|c|c|c|c|c|c|}
\hline 离 & 告 & 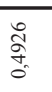 & 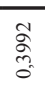 & 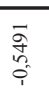 & $\begin{array}{l}\infty \\
\frac{\infty}{2} \\
\hat{a}_{3}^{2}\end{array}$ & & & & \\
\hline 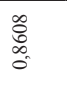 & $\begin{array}{l}0 \\
\frac{9}{0} \\
0\end{array}$ & 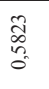 & $\begin{array}{l}0 \\
\text { o. } \\
0 \\
0 \\
0\end{array}$ & 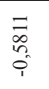 & $\begin{array}{l}\text { Fै } \\
\text { ôn } \\
0\end{array}$ & & & & $\begin{array}{l}\text { \$ิ } \\
\text { : }\end{array}$ \\
\hline 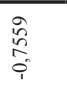 & $\begin{array}{l}\text { Tे } \\
\text { 尊 } \\
0\end{array}$ & 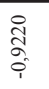 & $\begin{array}{l}\text { o } \\
\text { ât } \\
0 \\
0\end{array}$ & & & & $\begin{array}{l}0 \\
\hat{0} \\
0 . \\
0\end{array}$ & & 表 \\
\hline \multirow[t]{2}{*}{ 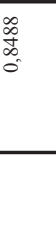 } & $\begin{array}{l}\text { बे } \\
\stackrel{0}{0} \\
0\end{array}$ & & & & $\begin{array}{l}\text { mat } \\
\text { f } \\
0 \\
0\end{array}$ & 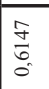 & 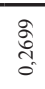 & & $\begin{array}{l}8 \\
\vdots \\
\infty \\
0\end{array}$ \\
\hline & & & $\begin{array}{l}\text { के } \\
\\
\end{array}$ & 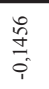 & $\begin{array}{l}\frac{\tilde{n}}{2} \\
\infty \\
0 \\
0\end{array}$ & $\begin{array}{l}\text { fo } \\
\text { qu } \\
\text { वे. }\end{array}$ & $\begin{array}{l}\text { 妾 } \\
\text { 它 }\end{array}$ & & $\frac{3}{5}$ \\
\hline 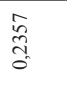 & $\begin{array}{l}\text { है } \\
\text { o. }\end{array}$ & 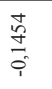 & $\begin{array}{l}\frac{n}{2} \\
\vdots \\
0 \\
0\end{array}$ & 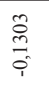 & 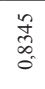 & 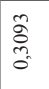 & 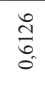 & & 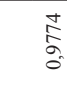 \\
\hline 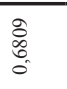 & 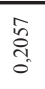 & 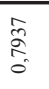 & $\begin{array}{l}\text { के } \\
\stackrel{0}{0} \\
0^{\circ}\end{array}$ & 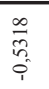 & 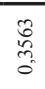 & 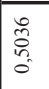 & $\begin{array}{l}\vec{F} \\
0 \\
0 \\
0\end{array}$ & & है: \\
\hline 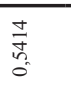 & $\begin{array}{l}0 \\
0 \\
0 \\
0 \\
0\end{array}$ & 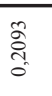 & 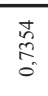 & $\begin{array}{l}\overrightarrow{0} \\
\overrightarrow{0} \\
\dot{i}\end{array}$ & 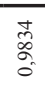 & 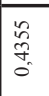 & 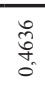 & & $\begin{array}{l}0 \\
\\
8\end{array}$ \\
\hline 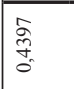 & $\begin{array}{l}\infty \\
\infty \\
q \\
0 \\
0\end{array}$ & 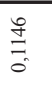 & 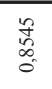 & 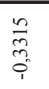 & $\begin{array}{l}\bar{r} \\
\hat{\infty} \\
0 \\
0\end{array}$ & 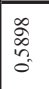 & $\begin{array}{l}\tilde{O} \\
\text { d్ } \\
\text { d. }\end{array}$ & & $\begin{array}{l}\text { त्: } \\
0\end{array}$ \\
\hline \begin{tabular}{|l|}
2 \\
2 \\
$\delta$ \\
0 \\
0
\end{tabular} & $\begin{array}{l} \\
0 \\
0 \\
0 \\
0\end{array}$ & 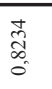 & $\begin{array}{l}\hat{0} \\
0 \\
0 \\
0\end{array}$ & 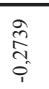 & $\begin{array}{l}\bar{n} \\
0 \\
0 \\
0\end{array}$ & 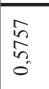 & $\begin{array}{l}\infty \\
\vdots 0 \\
0 \\
0 \\
0\end{array}$ & & $\begin{array}{l}\text { 志 } \\
\text { f } \\
0\end{array}$ \\
\hline 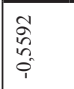 & $\begin{array}{l}\vec{N} \\
\text { d. } \\
0\end{array}$ & 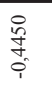 & 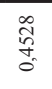 & 卓 & 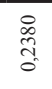 & 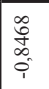 & $\begin{array}{l}0 \\
0 \\
0 \\
0 \\
0\end{array}$ & & $\begin{array}{l}\text { Oे } \\
\text { s. } \\
0\end{array}$ \\
\hline $\begin{array}{l}\vec{\sigma} \\
\text { o. } \\
0 \\
0\end{array}$ & 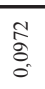 & $\begin{array}{c}\tilde{D} \\
\infty \\
\infty \\
0 \\
0 \\
0\end{array}$ & $\begin{array}{l} \\
\vdots \\
0 \\
0 \\
0\end{array}$ & 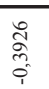 & $\begin{array}{l}0 \\
0 \\
0 \\
0 \\
0\end{array}$ & $\begin{array}{l}0 \\
0 \\
0 \\
0 \\
0 \\
0\end{array}$ & $\begin{array}{l}\text { ते } \\
\text { ปे }\end{array}$ & & $\begin{array}{l} \\
\text { 공 }\end{array}$ \\
\hline 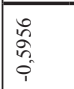 & 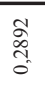 & 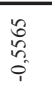 & 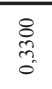 & 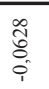 & 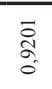 & 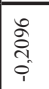 & $\begin{array}{l}\vec{n} \\
\hat{0} \\
0\end{array}$ & & $\frac{8}{0}$ \\
\hline $\begin{array}{l}\text { ta } \\
0 \\
0 \\
0 \\
i \\
i\end{array}$ & 叕 & & 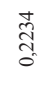 & 兽 & $\begin{array}{l}\bar{\alpha} \\
\infty \\
o \\
0\end{array}$ & 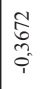 & 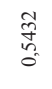 & & $\stackrel{\infty}{\bar{z}}$ \\
\hline 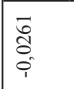 & $\begin{array}{l}\text { 总 } \\
\text { : } \\
0\end{array}$ & & $\begin{array}{l}0 \\
\text { 今. } \\
\infty \\
0 \\
0\end{array}$ & 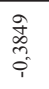 & तु & & 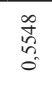 & & 孛 \\
\hline 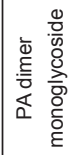 & & 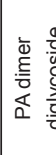 & & 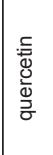 & & 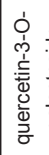 & & & 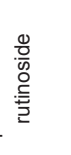 \\
\hline
\end{tabular}

